\title{
دراسة اقتصادية لصادرات مصر من الخرشوف
}

\author{
رقية حسن جبر، رويدا أسامة عويضة \\ قسم الاقتصاد الزر اعي - كلية الزر اعة- جامعة الزقازيق - مصر عردانه \\ الملخص
}

استهدف البحث دراسة تطور المساحة والإنتاج والإتتاجية للخرشوف، وتطور الصادرات الصصرية منه و التوزيع الجغر افي لها، وتقدير

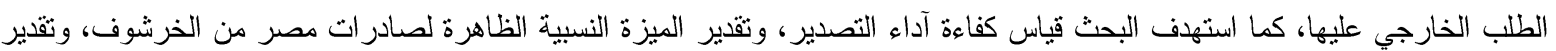

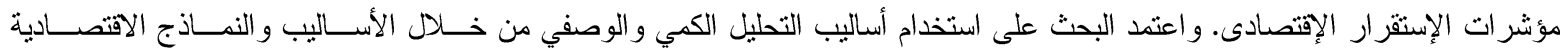

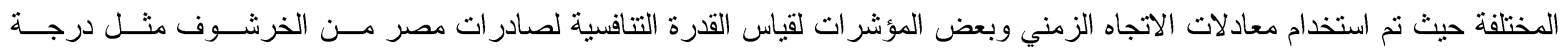

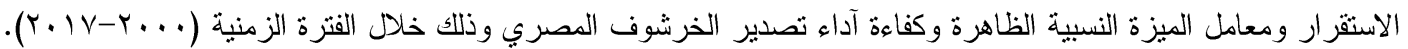

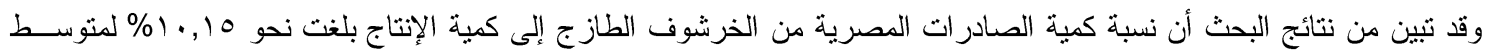

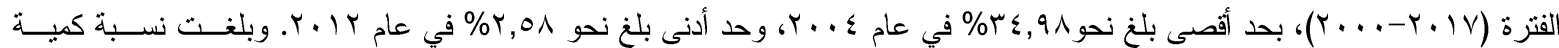

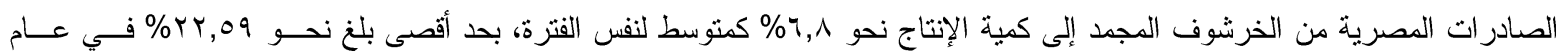

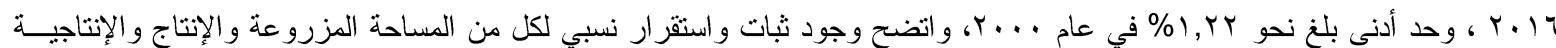

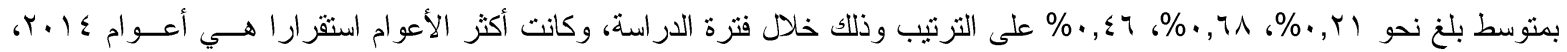

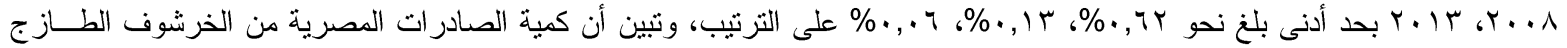
أكثر استقرارا من الخرشوف المجمد. كما تيين أن مصر تثتع بميزة نسبية ظاهرية لصادرات الخرشوف الطازج والمجمد بالعالم، حيث بلغ معامل الميزة النسبية الظاهرة

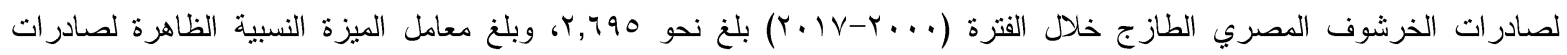
الخرشوف المصري المجمد خلال نفس الفترة نحو VYr, I. كذلك اتضح ثركز الصادرات المصرية من الخرشوف الطازج خلال الفترة

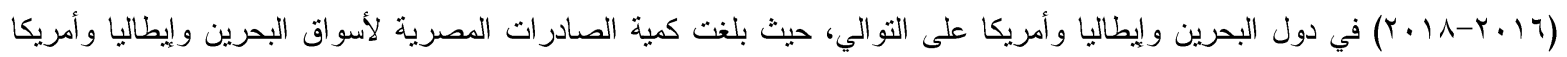

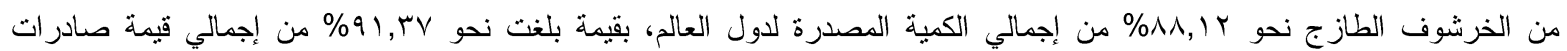
الخرشوف المصري لدول العالم. كما اتضح تركز الصادرات المصرية من الخرشوف المجمد خلال نفس الفترة في دول إيطاليا وأسبانيا

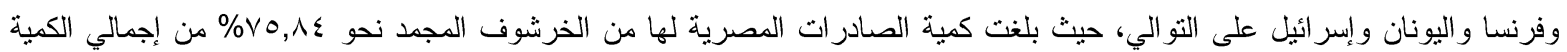

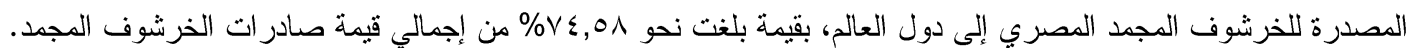
الكلمات الإفتتاحية: دراسة اقتصادية - صادرات - الخرشوف.

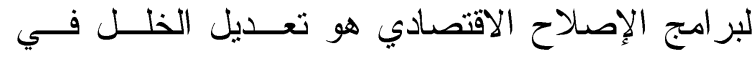

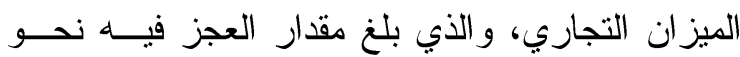

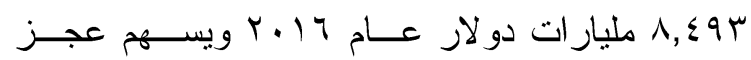

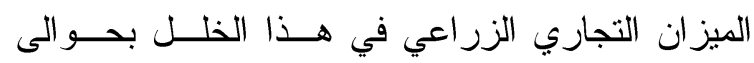

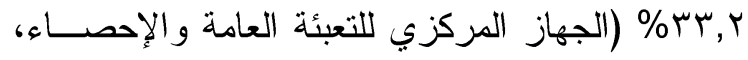

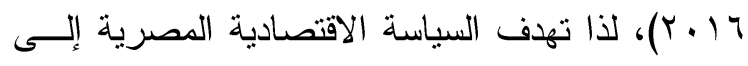

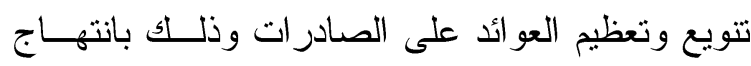

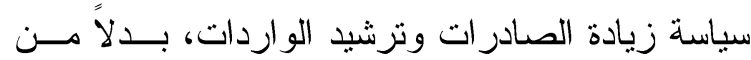
التزكيز على اتباع سياسة الإحلال محل الواردات.

\section{المقدمة و المشكلة البحثية}

يلعب قطاع التجارة الخارجيــة دوراً هامـــاً فـى لـى

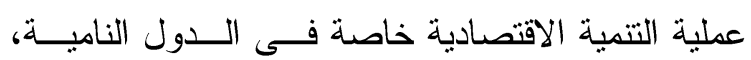
وتعتبر الصادرات من أهم العوامل التي تساهم فى زيادة

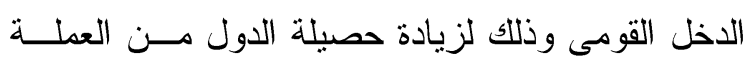

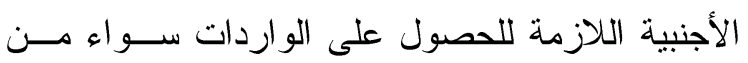

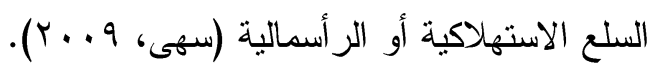

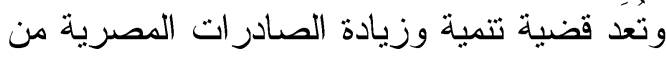
القضايا المحورية الهامة لأن أحد الأهــداف الرئيسـية 
تكمن مشكلة الدراسة في انخفاض الصادرات المصرية من الخرشوف مع عدم استقرارها حيث

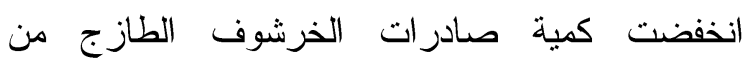

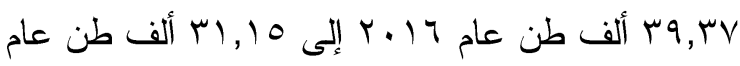

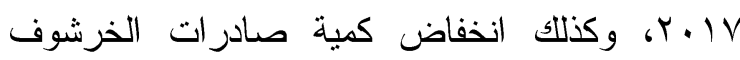

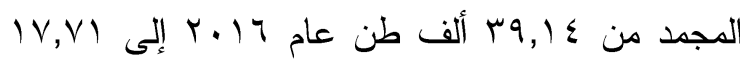

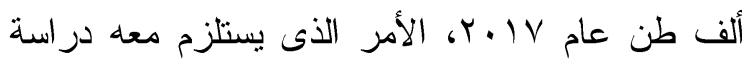
الأسواق العالمية للخرشوف وات القدرات التتافسية التصديرية المصرية لها.

\section{أهداف البحث}

ا. دراسة تطور المساحة و الإنتاج و الإنتاجية

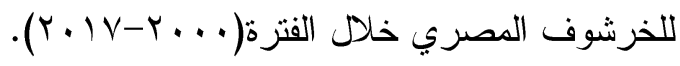

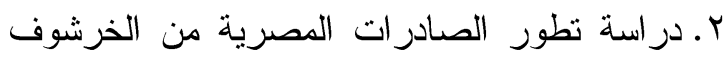

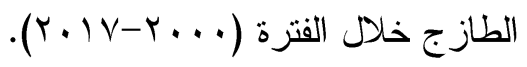

r. دراسة نطور الصادرات المصرية من الخرشوف

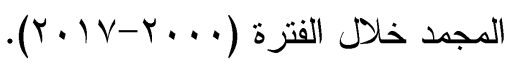

ع. تقدير مؤشرات الإستقرار الإقتصادى للخرشوف

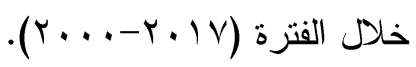

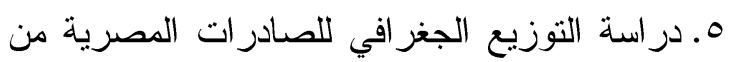

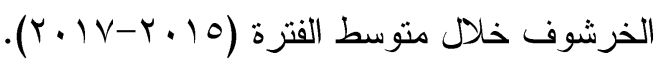
7 ـ نقدير الميزة النسبية الظاهرة لصادرات الخرشوف

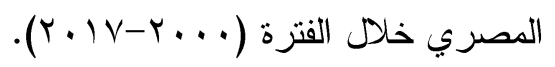

V. قياس كفاءة آداء تصدير الخرشوف المصري خلال

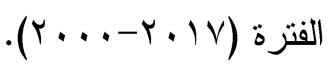

^. تقدير الطلب الخارجي على الصادرات المصرية

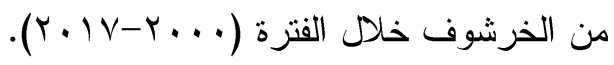

\section{الطريقة البحثية ومصادر البيانات}

اعتمد البحث على البيانات الثانوية المنشورة وغير المنشورة، والصادرة من كل من الجهاز المركزي للتعبئة العامة والإحصاء وقاعدة ولماءة بيانات الحاسب الآلي التابعة للجهاز، و الإدارة المركزية للاقتصاد الزراعي بوزارة الزراعة، ومنظمة الأغذية

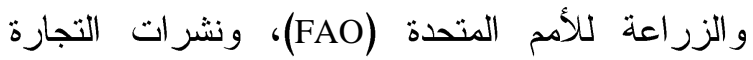

ويعتبر عــدم اســتقرار الصــادرات الزراعيــة

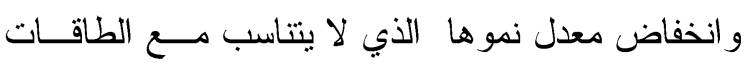

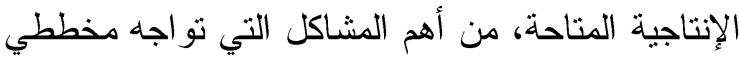

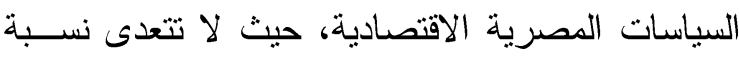

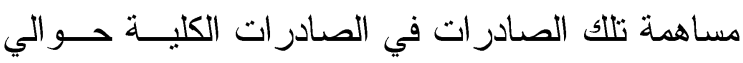

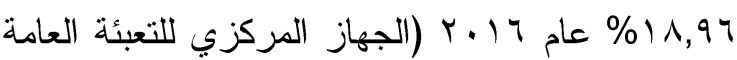

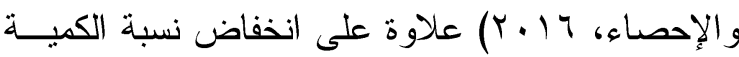

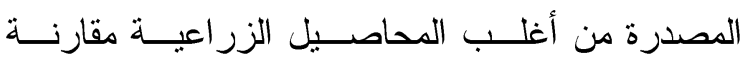

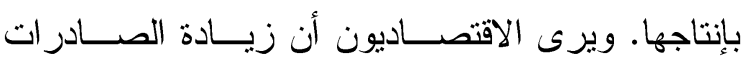
الزراعية تعتبر من أهم الأدوات الرئيسية لعلاج العجز

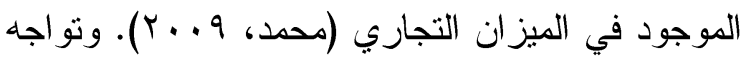

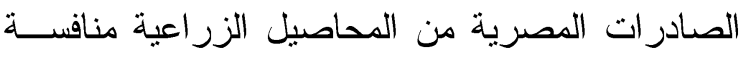
شديدة في الأسواق العالمية في ظل التكتلات الاقتصادية

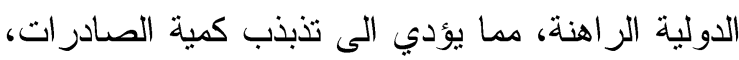
وبالتالي العجز في الميزان التجاري (سهام، • ( • ب). الخرشوف نبات عشبى معدــر، ويســتهلك مــن فئن

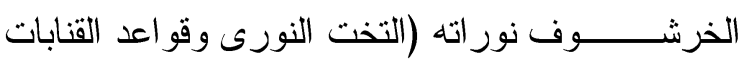
المحيطة بالنورة خاصة الداخلية منها) وتنتخدم الأوراق

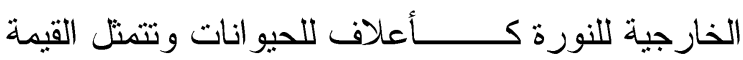
الغذائية للخرشوف في إحتو ائه على العديد من العناصر الغذائية الهامة و الكربو هيدرات مثل الحديد و الكالســــيوم و الفوسفور حيـث يحتل المركز السابع في الترتيب بين

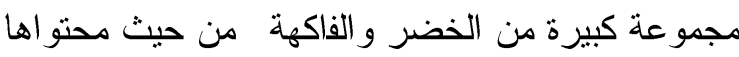
من عشرة فيتامينات ومعادن. يتجدد نمو الخرشوف كله سنويا بتكوين خلفات جديــدة في فصل الخريف، وتجدد زر اعة الخرشوف في مصر سنويا، بينما تجدد زر اعته

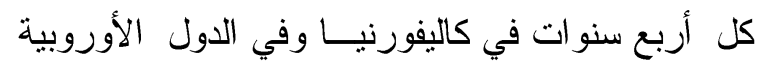

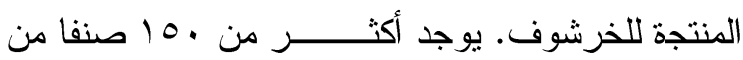

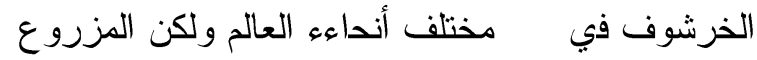

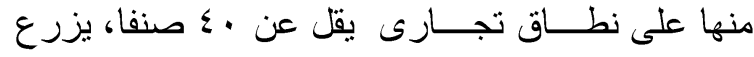
الخرشوف فى مصـر للتصدير بصفة أساسية ومن أهم الصناف التى تنتشر زراعتها في مصر هي الخرشوف البلدي، و الفرنساوي و الإيطالي (شيماء V... Y). 
يتبين أن المساحة المزروعة بالخرشوف تزاوحت

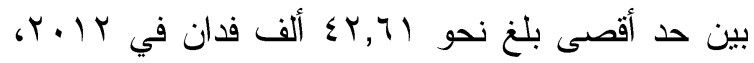

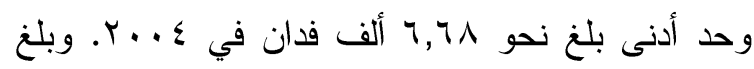

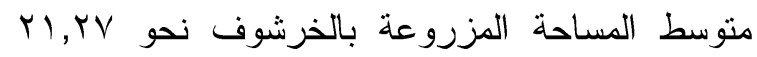

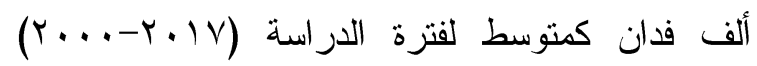
بمتوسط معدل تغير سنوي حو الي VV,V بزيادة معنوية

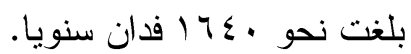
r- تطور الإنتاجية من الخرشوف المصري:

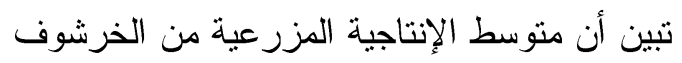

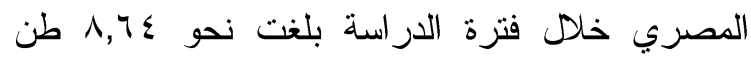
للفدان تراوحت بين حد أقصى بلغ بس, • ا طن للفدان

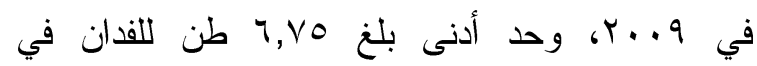

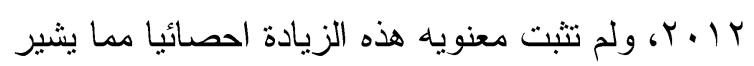
إلى الثبات النسبي حول المتوسط.
الخارجية، وبعض الدراسات السابقة في هذا المجال. و اعتمد البحث على استخدام أساليب التحليل الكمي لهي و الوصفي من خلال الأساليب و النماذج الاقتصادية المختلفة حيث تم استخدام معادلات الاتجاه الزمني وبعض المؤشرات لقياس القدرة التتافسية لصادرات مصر من الخرشوف مثل درجة الاستقرار ومعامل

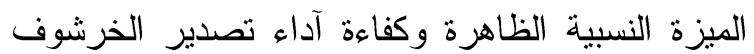

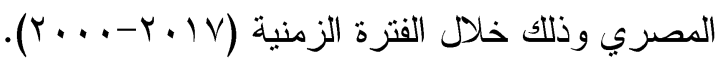

\section{النتئاج البحثية ومناقشتها}

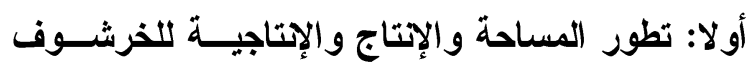

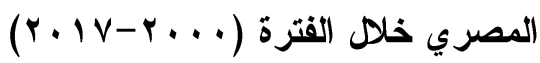

أوضحت النتائج الواردة بالجدولين (1)، (r) ما يلى:

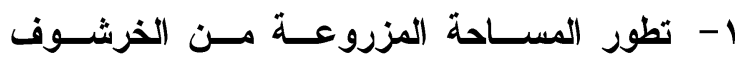

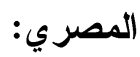

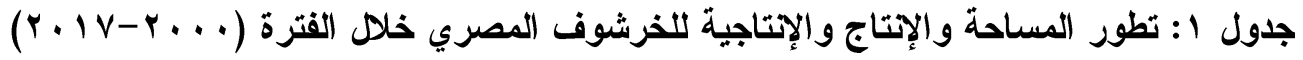

\begin{tabular}{|c|c|c|c|}
\hline الإتتاج (ألف طن) & الإتتاجية & المساحة (ألف فدان) & السنوات \\
\hline$\Lambda \vee, 9 \vee$ & $\Lambda, \cdot 9$ & $1 \cdot, \lambda V$ & r... \\
\hline $70, Y Y$ & $V, \wedge Y$ & $\Lambda, r_{0}$ & $r \ldots$ \\
\hline $71, \cdot r$ & 1,19 & $V, \leqslant 0$ & $r \ldots r$ \\
\hline $11 \cdot, r_{0}$ & $1, .0$ & $|r, v|$ & $r \ldots r$ \\
\hline $0 \leqslant, 01$ & 1,17 & 7,71 & $r \ldots \varepsilon$ \\
\hline $1 \wedge r, 90$ & $9, \varepsilon r$ & $19, \varepsilon 1$ & r...o \\
\hline$|r V, \Lambda|$ & $\Lambda, Y \varepsilon$ & $10,0 Y$ & $r \ldots r$ \\
\hline IVY,V. & $9, \cdot V$ & $19, .0$ & Y...V \\
\hline$I V T, r V$ & $9,7 \mathrm{~V}$ & $1 \lambda, r \leqslant$ & $r \ldots \lambda$ \\
\hline rol,10 & $1 ., 14$ & $r \varepsilon, r Y$ & $r \ldots q$ \\
\hline r10,0r & $1 ., 17$ & $r,, r$ & $r .1$. \\
\hline$Y \cdot Y, T \Sigma$ & $\Lambda, 9 \Lambda$ & YY,OY & $r .11$ \\
\hline$r \wedge \vee, V$. & 7,10 & $\{r, T)$ & $r \cdot 1 r$ \\
\hline$r \cdot \varepsilon, .$. & $\Lambda, \vee \uparrow$ & $r \varepsilon, v)$ & $r \cdot 1 r$ \\
\hline YTY,Y. & $\Lambda, \wedge \varepsilon$ & $r \cdot, 11$ & $Y+1 \varepsilon$ \\
\hline$r r_{,}, V_{O}$ & 1,70 & YO,OY & $r .10$ \\
\hline$|V r, r|$ & $\Lambda, \cdot \varepsilon$ & $r 1,00$ & $r .17$ \\
\hline$r r q, r$. & $\Lambda, r V$ & $\varepsilon 1, \cdot r$ & $r .1 \mathrm{~V}$ \\
\hline INT,r & $\Lambda, 7 \varepsilon$ & rl, rV & المتوسط \\
\hline
\end{tabular}

المصدر: الجهاز المركزى للتعبئة العامة والأحصاء، مركز المعلومات ودعم اتخاذ القرار، قاعدة بيانات التجارة الخارجية، القاهرة. 
جدول r: معادلات الإتجاه الزمنى العام لتطور المساحة والإتتاج والإتتاجية للخرشوف المصري خلا الفترة

$(r+\mid v-r+\cdots)$

\begin{tabular}{|c|c|c|c|c|}
\hline Fc & $\mathbf{R}^{2}$ & التغير السنوي" معل & صهـ= = أ+ب سعادلة سهـ & المتغيرات \\
\hline **Tr,T V & $\cdot, 7 \Lambda$ & $V, V)$ & 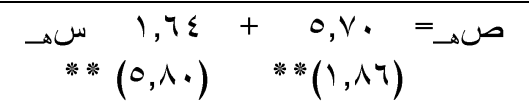 & المساحة المزروعة (ألف فدان) \\
\hline$* * r q, 1 \vee$ & $\cdot, \times 1$ & $V, \leqslant 0$ & 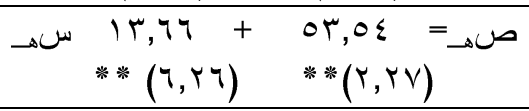 & الإنتاج (ألف طن) \\
\hline$\cdot, 10$ & $\cdot, \cdot 1$ & Tr, & 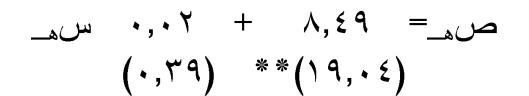 & الإنتاجية \\
\hline
\end{tabular}

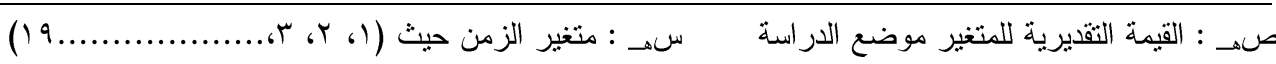
القيم ما بين الأقو اس = (ت المحسوبة).

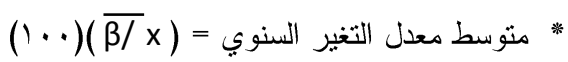
المصدر : حسبت بو اسطة برنامج التحليل الإحصائى (SPSS ver. 25) باستخدام بيانات الجدول (1) (1).

حوالي rی,9\%\% من متوسط الكمية المصدرة التي بلغت نحو ع •, ا ألف طن خلال فترة الدراسة، بينما بلغت

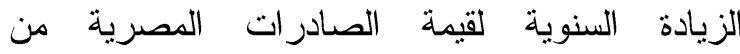
الخرشوف الطازج نحو عب,ا مليون دولار تمثل

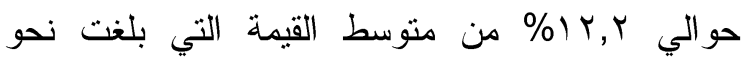
991, •1 مليون دولار خلال نفس الفترة. وتأكدت معنوية هذه الزيادة احصائيا عند مستوى معنوية

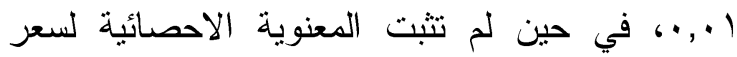
تصدير الخرشوف المصري الطازج مما يعني أنه

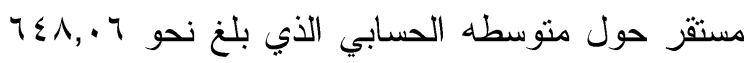
دو لار للطن خلال فترة الدراسة.

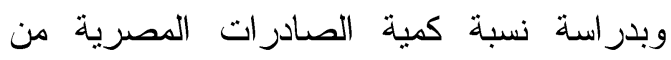

الخرشوف الطازج إلى كمية الإنتاج اتضح أنها

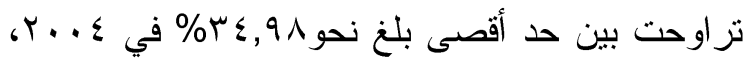

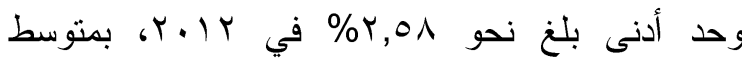

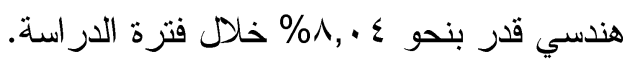
ثالثا: تطور الصادرات المصرية من الخرشوف المجمد بـا

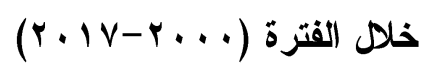

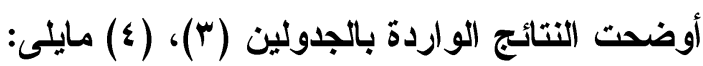
تزاوحت كمية صادرات مصر من الخرشوف بالجن

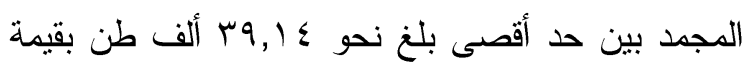

r- تطور الإنتاج من الخرشوف المصري:

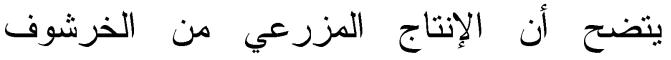

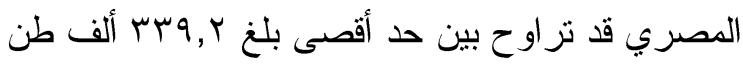

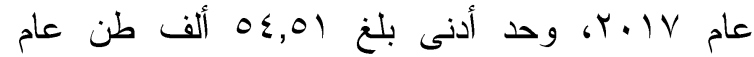
؟....، وقد تزايد الإنتاج المزرعي من محصول الخرشوف المصري سنويا بحوالى

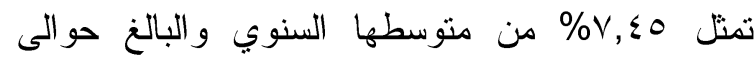

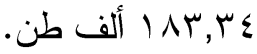
ثانيا: تطور الصادرات المصرية من الخرشوف الطازج

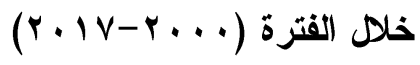

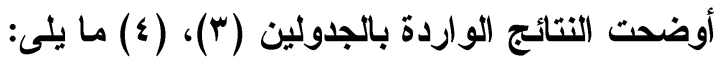

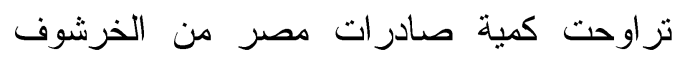

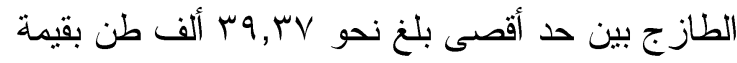

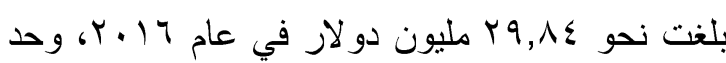

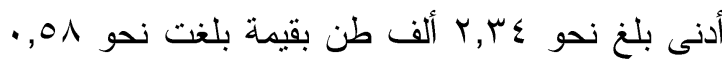

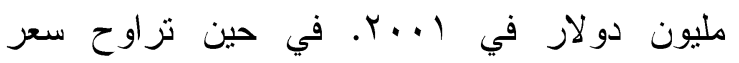
التصدير المصري للخرشوف الطازح بين حد أقصى في فين

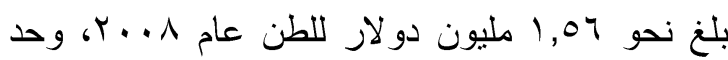

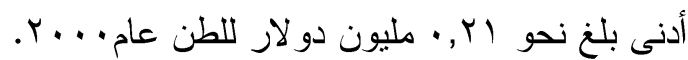
قدرت الزيادة السنوية لكمية الصادرات المصرية من الخرشوف الطازج بنحو آTV, ألف طن تمثل 
الفترة. في حين لم تثبت المعنوية الاحصائية لسعر تصدير الخرشوف المصري المجمد مما يعني أنه

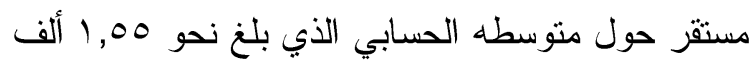
دو لار للطن خلال فتزة الدر اسة.

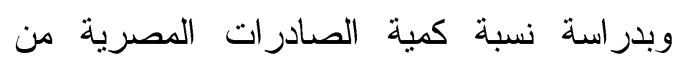
الخرشوف المجمد إلى كمية الإنتاج اتضح من الجدول

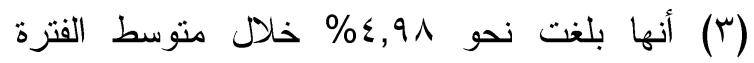
(Y. V-r...)

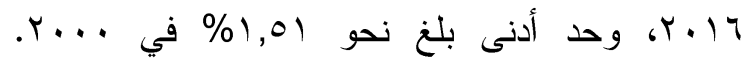

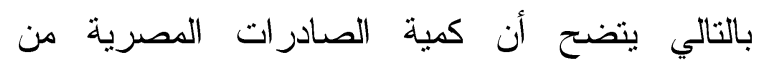
الخرشوف الطازج بالنسبة لكمية الإنتاج أكبر من نسبة لئة كمية الصادرات المصرية من الخرشوف المجمد لكمية
بلغت نحو 07,07 •T مليون دولار في عام 17 • ب، وحد

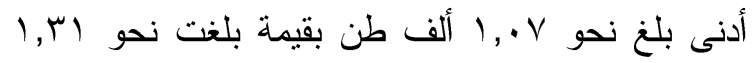

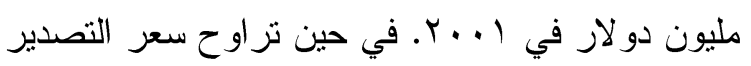

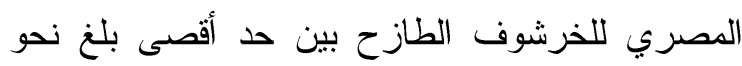

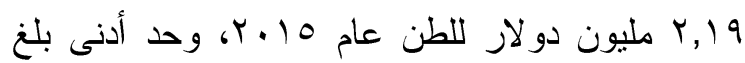

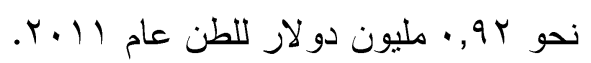
بتقدير الزيادة السنوية لكمية الصادرات المصرية من الخرشوف المجمد اتضحح ثبوت معنويتها احصائيا

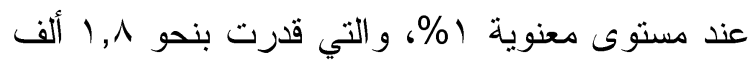

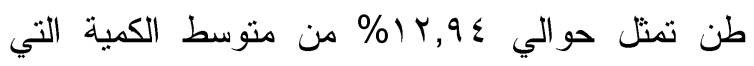

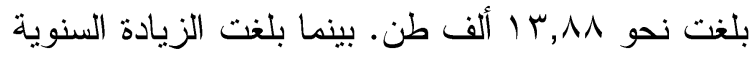
لقيمة الصادرات المصرية من الخرشوف المجمد نحو لبردي

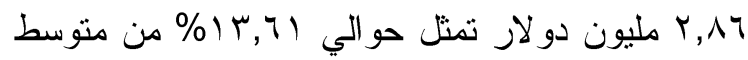

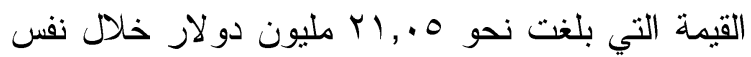

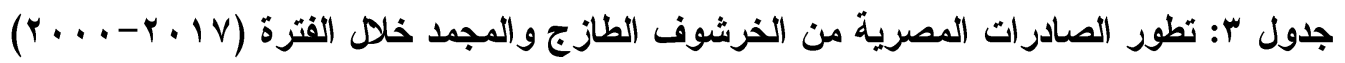

\begin{tabular}{|c|c|c|c|c|c|c|c|c|}
\hline \multicolumn{2}{|c|}{ الخرشوف/الإنتاج(\%) } & \multicolumn{3}{|c|}{ الخرشوف المجمد } & \multicolumn{3}{|c|}{ الخرشوف الطازج } & \multirow{3}{*}{ السنوات } \\
\hline \multirow{2}{*}{ المجمد } & \multirow[b]{2}{*}{ الطازج } & السعر & قيمة & كمية & السعر & قيمة & ية & \\
\hline & & (دولار للطن) & (مليون دولار) & (ألف طن & (دولار للطن) & (مليون دولار) & (ألف طن) & \\
\hline 1,01 & $\Lambda, 1 Y$ & $I Y V \wedge, Y$. & $1, v$. & נז & $r \cdot \wedge, \uparrow \wedge$ & $1, \leqslant 9$ & $V, 1 \leq$ & r... \\
\hline $1, T \leqslant$ & $r, 0 q$ & IYY $\{, r$. & $1, r)$ & $1, \cdot v$ & $r \leqslant V, \Lambda T$ & $\cdot, 01$ & $r, r \varepsilon$ & $r \ldots l$ \\
\hline$r, \xi 1$ & $0, V \leqslant$ & $10 \Omega \varepsilon, Y Y$ & $Y, Y V$ & $1, \varepsilon V$ & rov, I $\leq$ & $1, r_{0}$ & $r, 0$. & $r \ldots r$ \\
\hline $1,9 \varepsilon$ & $0, v \varepsilon$ & $1 \leqslant r \leqslant, 0 \wedge$ & $r, \cdot v$ & $Y, 1 \leq$ & YAY,VA & 1,199 & ד & $r \ldots r$ \\
\hline $0, r q$ & $r \varepsilon, 9 \wedge$ & $\mid \wedge \wedge \vee, V T$ & 0,00 & $r, q \varepsilon$ & rqV,rr & $0,7 \mathrm{~V}$ & $19, \cdot V$ & r.. \\
\hline $1,0 \mathrm{r}$ & 7,01 & $|V| \varepsilon, Y q$ & $\varepsilon, \wedge$. & $r, \Lambda$. & $V \Psi \varepsilon, \ldots$ & $\Lambda, \wedge \Gamma$ & $1 Y, \cdot r$ & $r \ldots o$ \\
\hline$r, 79$ & $1 \cdot, \pi$ & TYVO, \&Y & $T, \cdot Y$ & $\varepsilon, V Y$ & $7 \vee 9,11$ & $9, r \pi$ & $1 \%, 09$ & T..T \\
\hline$\Gamma, 1 \wedge$ & $\varepsilon, r \mu$ & $1 \pi v 1,09$ & $V, O T$ & $0, \leqslant 9$ & $A Y \cdot, T r$ & $7,1 \pi$ & $V, \sum V$ & Y..V \\
\hline 7,97 & $r, 74$ & $1 \leqslant Y Y, T \leqslant$ & $\mid V, \Sigma V$ & $\mid Y, Y A$ & $1071, \leqslant 9$ & $1 \cdot, \cdot 1$ & $7, \leqslant 7$ & $r \ldots A$ \\
\hline$V, 9 \mu$ & $\Lambda, 乏)$ & $10 \sum V, r Y$ & $r \cdot, q$. & $19,9 \mathrm{~V}$ & IYYY,T\& & $r 0,99$ & $r 1,19$ & r.. \\
\hline $11,7$. & $11,7$. & 10YV,T. & $r \lambda, 19$ & ro,.. & rqv,... & 9,94 & $r_{0}, \ldots$ & $r \cdot 1$. \\
\hline $1 \Lambda, r$. & $17, \vee \wedge$ & $910,7 \mathrm{~V}$ & $r T, V V$ & $r 4, \wedge \wedge$ & $M 11, \varepsilon V$ & $1 \cdot, 09$ & $r \varepsilon, .$. & r.11 \\
\hline$v, v \varepsilon$ & $r, 0 \Lambda$ & YIY T,OV & $\varepsilon \vee, r \Lambda$ & YY,YA & $1.70, \leqslant \varepsilon$ & $v, 9)$ & $V, \varepsilon r$ & $Y . I Y$ \\
\hline $0, .0$ & $\varepsilon, 10$ & $Y .09,9 \varepsilon$ & r1, Tr & 10,40 & V)r,.o & $9, \ldots$ & איד, & $r .1 T$ \\
\hline $9, \vee \wedge$ & 11,10 & $1 \ldots r, \leqslant \tau$ & $Y \Psi, Y$ & $r 7, \cdot r$ & $r \cdot 0, \Lambda r$ & $9, \cdot 1$ & $r q, \uparrow \wedge$ & $Y \cdot 1 \varepsilon$ \\
\hline 0,99 & $M, \wedge \varepsilon$ & Y194,0. & $r q, \cdot r$ & $M, Y T$ & 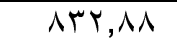 & Tr,T & rA, ro & $r .10$ \\
\hline$Y Y, O \Lambda$ & $Y Y, V Y$ & $10 \leqslant V, Y V$ & $7 ., 07$ & $r q, 1 \leq$ & $v \circ V, q$. & $r q, \wedge \varepsilon$ & $r q, r v$ & $r .17$ \\
\hline $0, Y Y$ & 9,11 & IVAT,IV & $\Gamma 1,01$ & $|V, V|$ & $\Delta T 0, \lambda)$ & $Y 7,9 V$ & $\Gamma, 10$ & T.IV \\
\hline$\varepsilon, 9 \Lambda *$ & $\Lambda, \cdot\{*$ & $10 \leqslant V, 7 \leqslant$ & $r 1, .0$ & $1 \mu, \wedge \Lambda$ & $T\{\wedge, \cdot T$ & $1 \cdot, 991$ & $I V, \cdot \varepsilon$ & المتوسط \\
\hline
\end{tabular}

المصدر: الجهاز المركزى للتعبئة العامة والأحصاء، مركز المعلومات ودعم اتخاذ القرار، قاعدة بيانات التجارة الخارجية ، القاهرة. 
جدول \&: معادلات الإتجاه الزمنى العام لتطور الصادرات المصرية من الخرشوف الطازج خلال الفترة (... بـ

$(r \cdot 1 \mathrm{~V}$

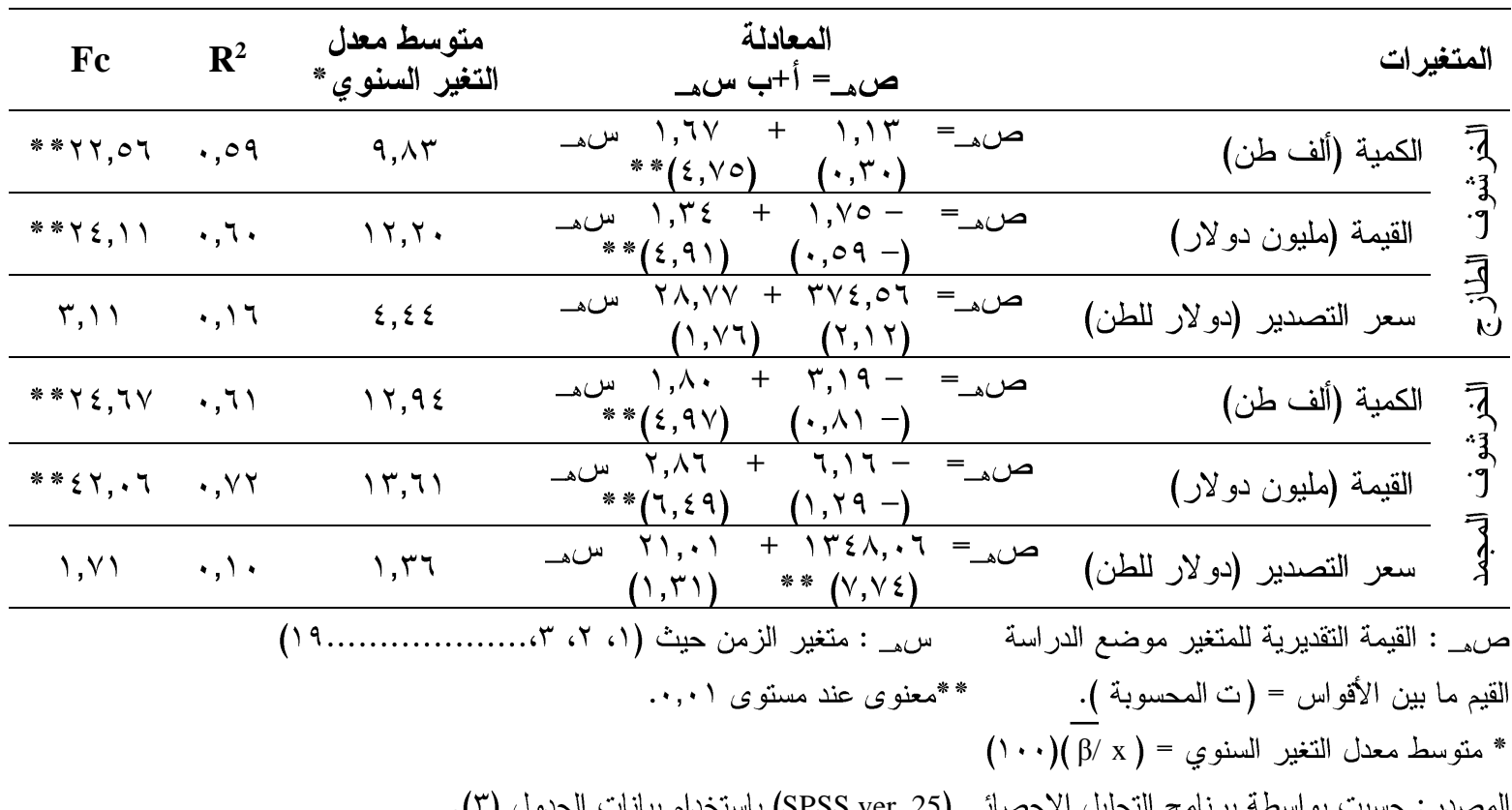

No معامل عدم الإستقر ار N.S

t القيمة الحقيقية الفعلية للمتغير المدروس فى السنة

القيمة الإتجاه الزمنى العام للظاهرة المدروسة الإنها

وقد تم تحليل هذا الاستقرار الاقتصادي من خلامل

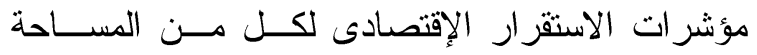

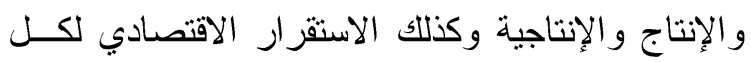

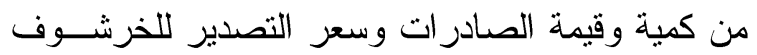

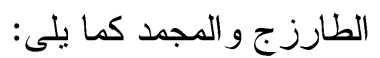
1- درجة الاستقرار للمساحة المزروعة والإنتاج والإتتاجية من الخرشوف:

بتقدير وتحليل درجة الاستقرار الاقتصادي لكل من من الإنئ المساحة المزروعة و إنتاج و إنتاجية الخرشوف في لئي مصر اتضح من الجدول (0) أن المتوسط العام لمؤشر أنس

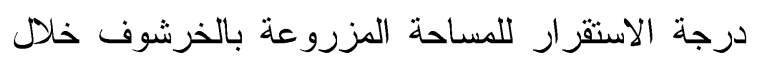

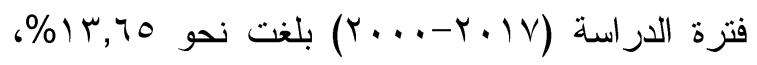
وتزاوحت قيمة معامل الاستقرار للمساحة المزروعة

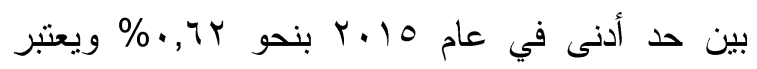
أكثر الأعو ام استقرارا، بينما بلغ حده الأقصى في عام
رابعا: تقدير مؤشرات الإستقرار الإقتصادى للخرشوف

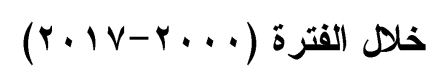

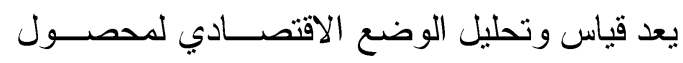

الخرشوف فى مصر سو اء من حيث المساحة والإنتــاج والإنتاجية أو من حيث كمية وقيمة الصـــادر ات خــلهل

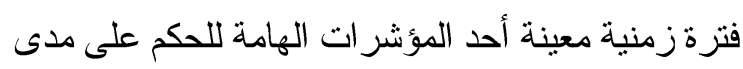
إستقرار السياسات الإنتاجية و التصديرية، و الوصول إلى لى لى مستوى معين من الاستقرار الاقتصادى يستهدف تحقيق

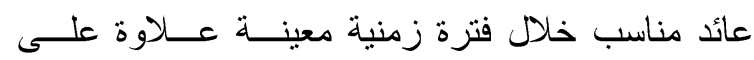
استقر ار هذا العائد، ويعتبر معامل عدم الاستقرار مــن فئن

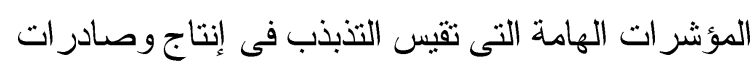
السلع المختلفة التى منها الخرشوف، وتشير قيمة معامل

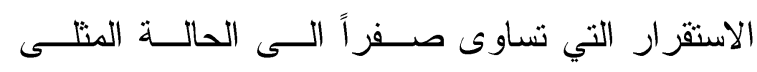
لاستثرار صـادرات السلعة، وكلما زادت قيمة المعامـلـل

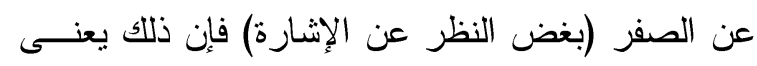
عدم الاستقر ار في الصــادرات (Porter, M. E, 1990). ويتم حساب معامل الاستقرار طبقا للمعادلة التالية: حيث أن: 
الاستقرار نحو 190,71\% وهو الحد الأقصى خلال فترة الدر اسة.

r- درجة الاستقرار لقمية الصادرات المصرية من الخرشوف:

يتضح من جدول(0) أن قيمة الصادرات المصرية

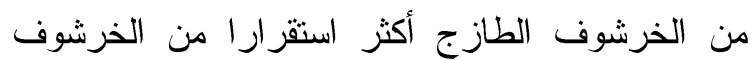
المجمد، حيث بلغ متوسط مؤشر درجة الاستقرار لقيمة

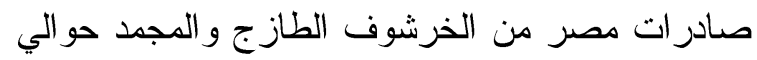
\%ץ\&,VO الدراسة. كانت أكثر الأعوام استقرارا من حيث قيمة

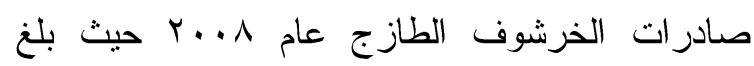

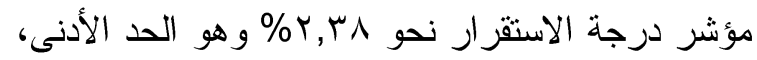

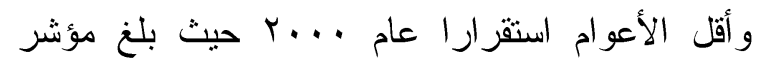

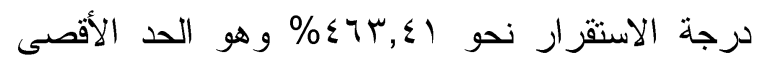

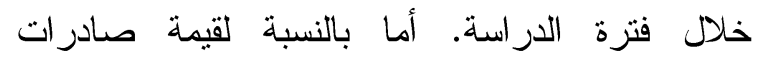
الخرشوف المجمد فكانت أكثر الأعوام استقرارا عام بـام

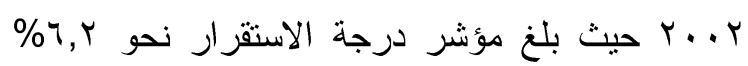

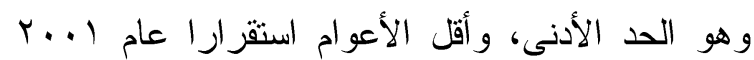

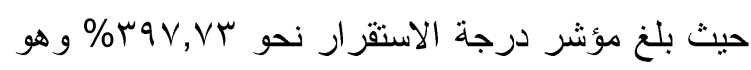
الحد الأقصى خلال فترة الدراسة.

ع- درجة الاستقرار لسعر الصادرات المصرية من فن لأندة

\section{الخرشوف:}

يتضح أيضا من الجدول (0) أن درجة الاستقزار

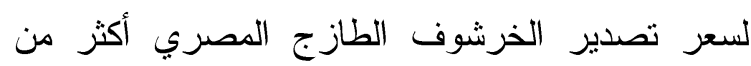
درجة استقرار سعر تصدير الخرشوف المجمد، حيث بلغ منوسط مؤشر درجة الاستقرار لكل منهما نحو

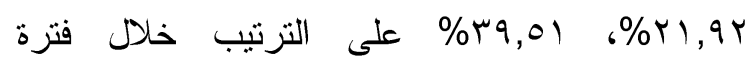

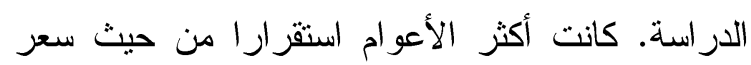

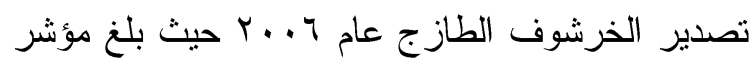

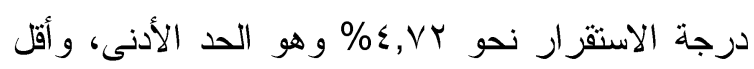

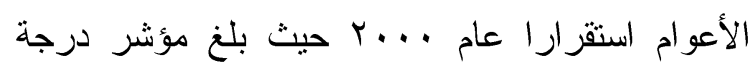

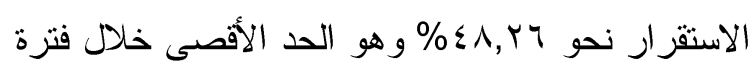

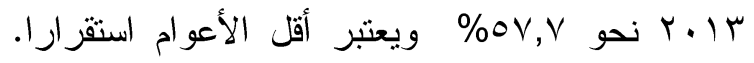
تنشير هذه النتائج إلى أن المساحة المزروعة بالخرشوف تتسم بالثبات و الاستقر ار النسبي خلال فترة الدراسة. بينما بلغ متوسط مؤشر درجة الاستقرار لإنتاج

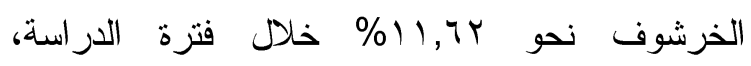

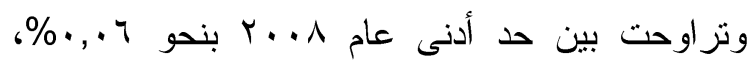

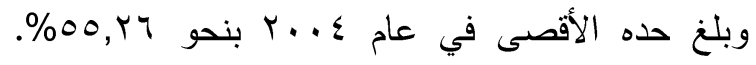
تنشير هذه النتائج إلى أن إنتاج الخرشوف ئن ينسم بالثبات و الاستقرار النسبي خلال فترة الدر اسة. أما بالنسبة لكتوسط مؤشر درجة الاستقرار

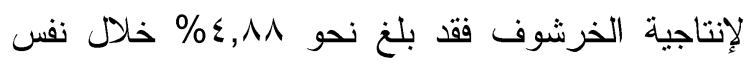

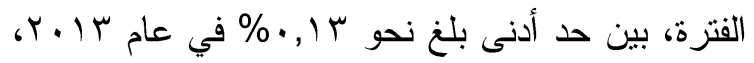

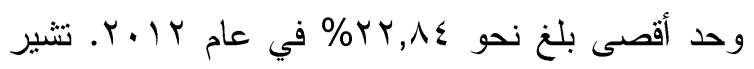

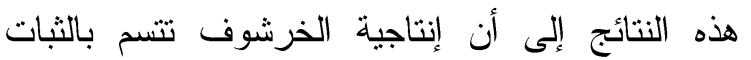
و الاستقرار النسبي خلال فترة الدراسة. r- درجة الاستقرار لكمية الصادرات المصرية من الخرشوف: بتقدير درجة الاستقرار لكل من كمية صادرات مصر من الخرشوف الطازج والخرشوف المجمد تبين

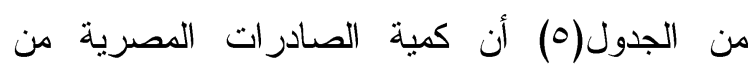
الخرشوف الطازج أكثر استقرارا عن كمية الصادرات

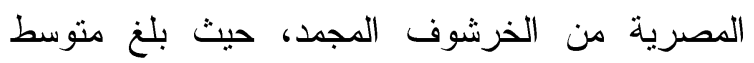

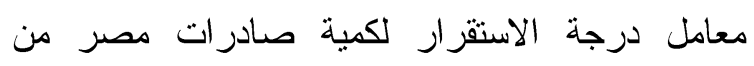
الخرشوف الطازج و المجمد خلال فترة الدراسة نحو

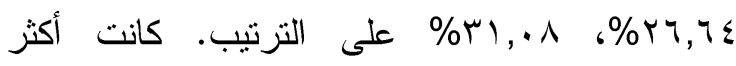

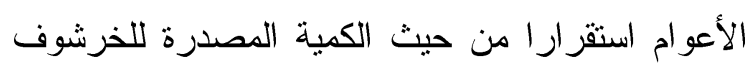
الطازج عام 10 . ب حيث بلغ مؤشر درجة الاستقرار

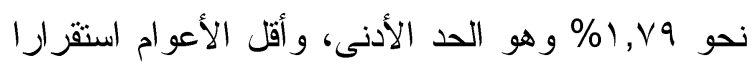

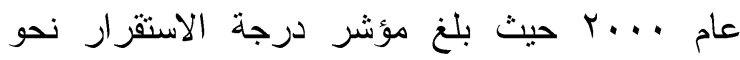

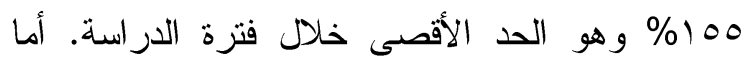
بالنسبة للكمية المصدرة من الخرشوف المجمد فكانت

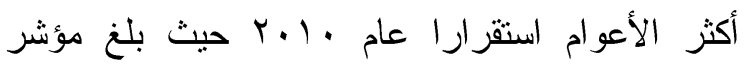

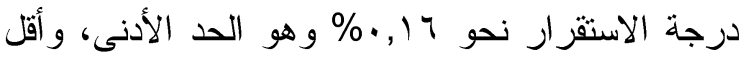

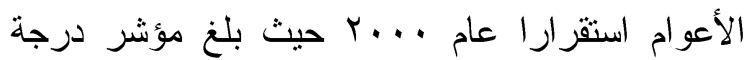




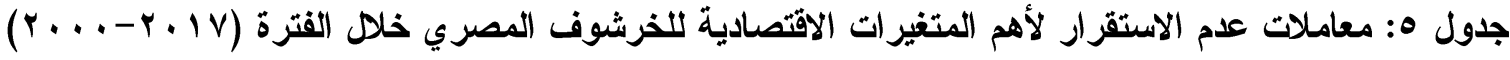

\begin{tabular}{|c|c|c|c|c|c|c|c|c|c|}
\hline \multicolumn{9}{|c|}{ معاملات عدم الاستقرار \% } & \multirow{3}{*}{ السنو ات } \\
\hline \multicolumn{3}{|c|}{ الخرشوف المجمد } & \multicolumn{3}{|c|}{ الخرشوف الطازج } & \multirow[b]{2}{*}{ الإتتاجية } & \multirow[b]{2}{*}{ الإتتاج } & \multirow{2}{*}{ المزروعة المساحة } & \\
\hline التصدير & القيمة & الكمية & التصدير & القيمة & الكمية & & & & \\
\hline $7,7 \leqslant$ & $101,0 Y$ & 190,71 & $\sum \Lambda, Y \uparrow$ & $\{\pi \mu, \varepsilon)$ & $100, \ldots$ & $\varepsilon, q$. & $r \cdot, 91$ & $\sum \lambda, \cdot 9$ & r... \\
\hline $11,9 \pi$ & $r q V, V T$ & $17 \cdot, 91$ & $\varepsilon Y, Y \leqslant$ & $r V, V V$ & $\varepsilon V, 10$ & $\Lambda, r_{\Lambda}$ & $19, \times 9$ & $V, \cdot r$ & r... \\
\hline $9, \varepsilon r$ & $7, Y$. & $r \mu, \Sigma \Lambda$ & YY,OI & $\varepsilon 0, \cdot r$ & $\varepsilon r, \ldots$ & $\varepsilon, Y$. & $r_{0, \varepsilon} \varepsilon$ & $r 9, \wedge 0$ & $r \ldots r$ \\
\hline$\cdot, \mathrm{IV}$ & $\varepsilon 1, \wedge \uparrow$ & $\sum 7,7 \pi$ & $\varepsilon Y, Y_{O}$ & $0 ., 0$. & 11,90 & $7, \cdot 1$ & $r, \cdot$, & $11, \lambda \Gamma$ & $r \ldots r$ \\
\hline$r 9,9)$ & $r, \Lambda r$ & $\varepsilon 9, \varepsilon$. & $\leqslant Y, 70$ & $1 \varepsilon, \pi$ & $1.1,17$ & $0, \ldots$ & $00, Y 4$ & $01,9 \varepsilon$ & r... \\
\hline 17,19 & $07, r 4$ & $\pi r, r)$ & $r \leqslant, 1 \leqslant$ & $\{\cdot, 1\}$ & $\vee, \wedge 9$ & $9, \sum V$ & $r o, \cdot r$ & $r \leqslant, q$. & r... \\
\hline 91,19 & $04,0 \mathrm{~V}$ & $T V, T V$ & $\varepsilon, V Y$ & $r \cdot, V \lambda$ & $7, \cdot 1$ & $\varepsilon, O \mathrm{~V}$ & $1 \leqslant, r \mid$ & 9,77 & r... \\
\hline $9 \vee, 9 r$ & $0 \leqslant, 97$ & $71, .7$ & $0, \leqslant 9$ & WI,VV & $\varepsilon \wedge, \leqslant 0$ & $\varepsilon, \wedge$. & $Y, \cdot V$ & $1, K Y$ & $r \ldots V$ \\
\hline $99, \cdot V$ & $1 \cdot, \mathrm{VA}$ & $r V, q r$ & $I Y, Y A$ & r, rی & $7 ., .0$ & 11,04 &.,+7 & $1 \cdot, 10$ & $r \ldots \lambda$ \\
\hline 99,19 & $r v, v$. & $1 \cdot, \mathrm{V} r$ & $19,9 V$ & IYY,VO & $\overline{l \Lambda, \lambda r}$ & $19,1 \mathrm{~V}$ & $r Y, \Sigma T$ & $1 ., .0$ & $r \ldots q$ \\
\hline $99, \leqslant 0$ & $0 ., 90$ &., 17 & ro, . & $r \Psi, T V$ & $r \Lambda, Y)$ & $17,7 \mathrm{~V}$ & $0, \sqrt{ } \uparrow$ & $1 ., 74$ & $r .1$. \\
\hline 99,74 & $19,9 Y$ & $r, \wedge V$ & $r, \wedge \wedge$ & $Y \Psi, Y)$ & $7 \cdot, 7$. & $r, \wedge 9$ & $I, \wedge Y$ & 11,11 & $r .11$ \\
\hline $99, Y$. & OY,V乏 & $r \neg, \cdot \wedge$ & YY,YA & $\leqslant 9,7$. & $T V, \Sigma \Lambda$ & $Y Y, \wedge \varepsilon$ & $r \varepsilon, \varepsilon \wedge$ & or,V. & $r .1 Y$ \\
\hline $9 \Lambda, \vee \vee 9$ & $7, T V$ & or,1. & 10,10 & $\varepsilon V, I V$ & $\varepsilon \wedge, \leqslant 9$ & $\cdot, 1 \mu$ & $r \varepsilon, 19$ & $r, 11$ & $r .14$ \\
\hline 99,19 & $r \wedge, q)$ & $r q, r$. & $r 4, \cdot r$ & $0 ., 09$ & וT, &., $0 \mathrm{~V}$ & $r, .$. & $\cdot ., T$ & r. $1 \varepsilon$ \\
\hline $99, .1$ & $Y T, V Y$ & $70,1$. & $1 \pi, r \pi$ & $19, \mathrm{Vr}$ & 1,19 & $1, \Lambda$. & $\backslash \wedge, \wedge \vee$ & $r \cdot, 1)$ & $r .10$ \\
\hline $99, \vee \leqslant$ & $\varepsilon r, T r$ & r, ז & $r q, 1 \leq$ & $\varepsilon 1, \uparrow \wedge$ & $r \mu, r v$ & $\lambda, 91$ & $r q, r_{0}$ & $\Gamma 0, \wedge \varepsilon$ & $r .17$ \\
\hline $9 \wedge, \wedge 9$ & $r \cdot, r Y$ & $0 \wedge, 9$. & $|v, v|$ & $r \cdot, \mu \Lambda$ & $\varepsilon, \cdot V$ & $7,0 \mathrm{~V}$ & $I r, Y \wedge$ & $17, \leqslant V$ & $Y . I V$ \\
\hline$r q, 01$ & rq,§) & $\mu, \cdot \wedge$ & $r 1,9 r$ & $\Gamma \varepsilon, V_{0}$ & $Y Y, Y \varepsilon$ & $\varepsilon, \wedge \wedge$ & $11,7 r$ & $1 \pi, 70$ & المتو سط" \\
\hline
\end{tabular}

• المتوسط السنوي للنسب المئوية تم حسابه كمتوسط هندسي.

المصدر : جمعت وحسبت بو اسطة برنامج النحليل الإحصائى (SPSS ver. 25) باستخدام بيانات الجداول (') و (r).

في المستقبل، وذلك من خلال نسبة نصيب الدولة من

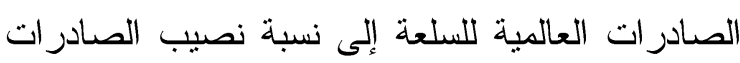
العالمية لتلك السلعة من الصادرات الزر اعبة العالمية. ويبين حساب المعامل إلى وجود ميزة نسبية إذا كانت قيمة المعامل أكبر من الواحد الصحيح، أما إذا كانت قيمته أقل من الواحد فإن ذلك يعني عدم وجود ميزة نسبية لتلك السلعة (Deardorff, A. V, 1984). ويتم حساب الرقم القياسي للميزة النسبية الظاهرة وفقاً

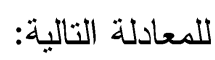

أما باللنسبة لسعر تصدير الخرشوف المجمد فكانت

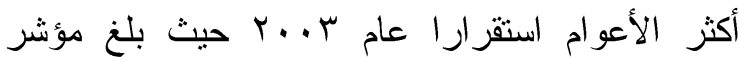

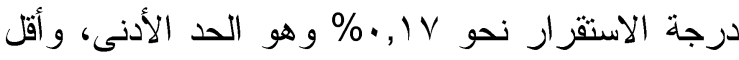

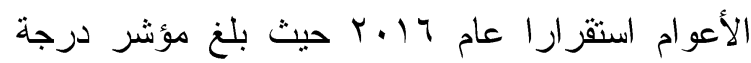

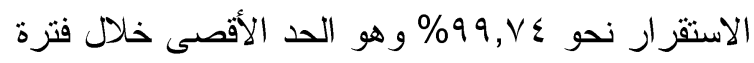

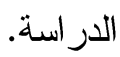

خامسا: الميزة النسبية الظاهرة لصادرات الخرشــوف

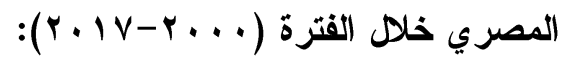

يشير معامل الميزة النسبية الظاهرة إلى الميزة التنافسية للصادرات من السلع، و الفرص المحتملة لها لهيرة الهيره

قيمة الصادرات الكلية للدولة / قيمة الصادرات الكلية العالمية

قيمة صادر ات السلعة للدولة / قيمة صادر ات السلعة في العالم 
متوسط مؤشر كفاءة آداء التصدير للخرشوف الطازج

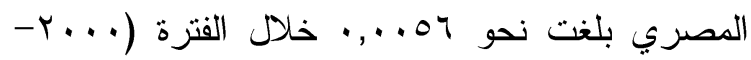

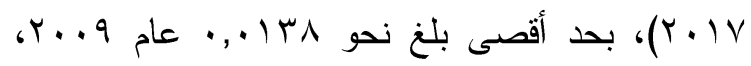

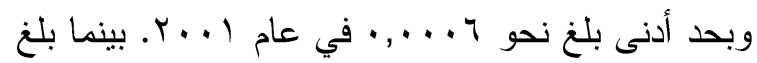

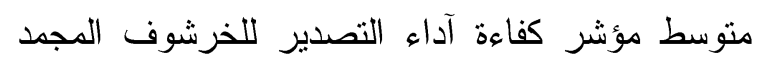

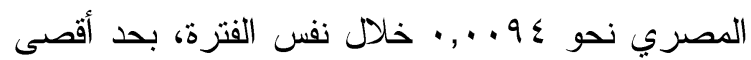

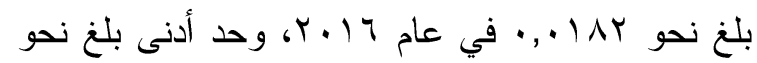

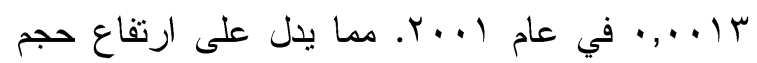

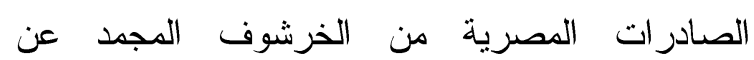
الخرشوف الطازج.

سابعا: التوزيع الجغرافي للصادرات المصرية من

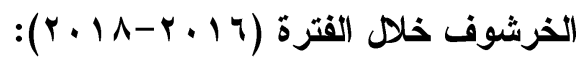

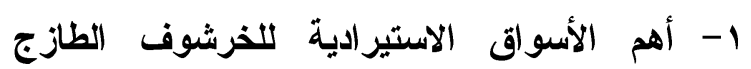

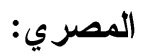

بدراسة التوزيع الجغرافي للصادرات المصرية من الخرشوف الطازج لدول العالم اتضح من الجدول تركز الصادرات المصرية من الخرشوف الطازج (V)

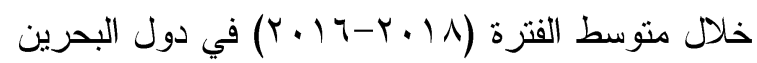

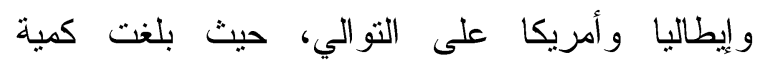
الصادرات المصرية لأسواق البحرين وإيطاليا و أمريكا

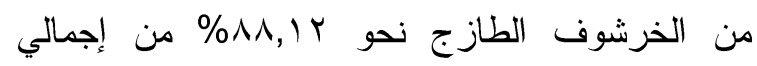

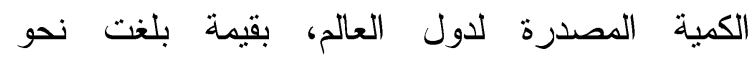
\% \% 1,rV المصري لدول العالم. يليها دول لبنان وريها وروسيا

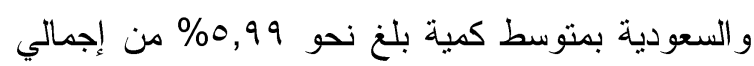
الكمية المصدرة من الخرشوف الطازج المصري، بقيمة

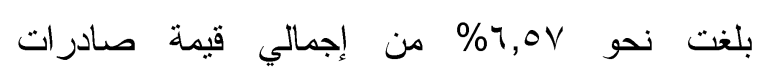
الخرشوف المصري لدول العالم خلال متوسط نفس الفتزة.

ويوضح الثكلين(1) و(Y) الأهمية النسبية لكميــة وقيمة الصادرات المصرية من الخرشــوف الطــازج

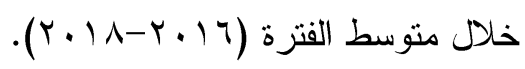

يتبين من الجدول(ا) أن متوسط معامل الميزة النسبية الظاهرة لصادرات الخرشوف المصري الطازج

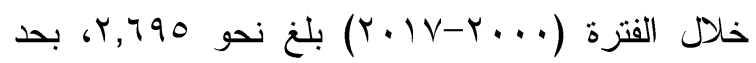

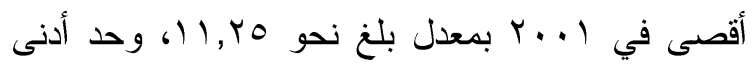

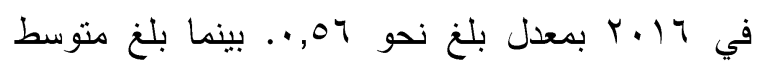

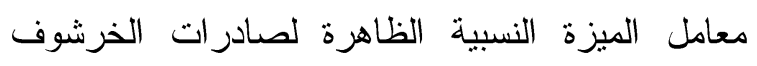
المصري المجمد خلال نفس الفترة نحو VY T T, ا، بحد

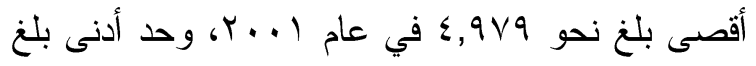

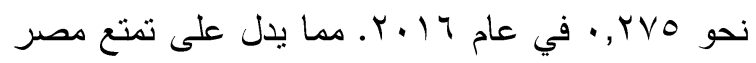
بميزة نسبية ظاهرية لصادرات الخرشوف الطازج و المجمد بالعالم وذلك لارتفاع قيمة المؤشر عن الواحد الصحيح خلال متوسط فترة الدر اسة. سادسا: كفاءة آداء تصدير الخرشوف المصري خلا (القترة (V) تؤثر كفاءة العمليات التصديرية على القدرة التنافسية للصادرات المصرية فى ضوء ضوء زيادة حدة المنافسة العالمية خاصة فى إطار الإتجاه نحو العالمية و التكتلات الإقليمية، وتتضمن كفاءة أداء العمليات

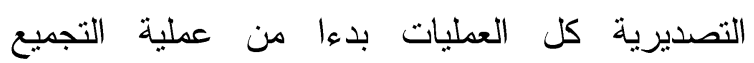

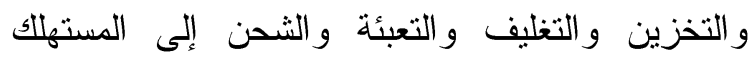

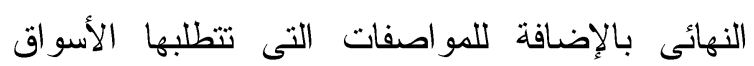
المختلفة و الإلتز ام بمعايير الجودة، وقوانين حماية البيئة.

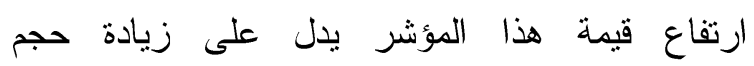

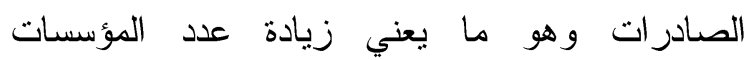
التصديرية (عاطف جودة، صلاح مقلد، 991 (1)). يمكن صياغتها وفقا للعلاقة التالية: $E t=\frac{X t}{G D P t} * 100$

حيث أن: t معيار كفاءة آداء العمليات التصديرية فى الفترة : Et t قيمة صادر ات الخرشوف فى الفترة : Xt

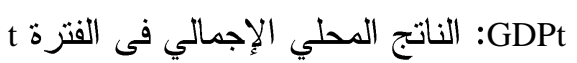
بتقدير مؤشر كفاءة آداء التصدير للخرشوف

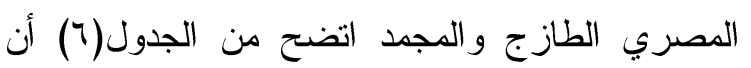




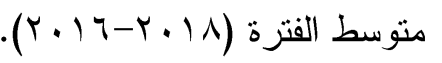
ويوضح شكل (r) و الثكل(ع) الأهمية النسبية لكمية وقيمة الصادرات المصرية من الخرشوف المجمد خلال

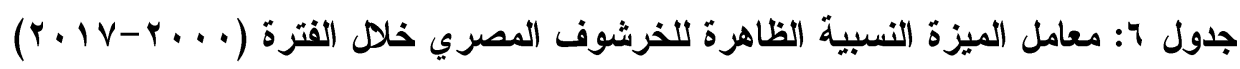

\begin{tabular}{|c|c|c|c|c|c|c|c|c|}
\hline \multicolumn{2}{|c|}{ معامل كفاعة آداء } & \multirow{2}{*}{ (الاخل القومي - } & \multicolumn{2}{|c|}{ معامل الميزة النسبية } & \multirow{2}{*}{ قايمة صادرات } & \multirow{2}{*}{ قيمة الصادرات } & \multirow{2}{*}{ قيمة الصادر ات الزراتية } & \multirow{2}{*}{ السنوات } \\
\hline الخرشوف & الخرشوف الطازج & & الخرشوف & الخرشوف الطازج & & & & \\
\hline$\cdot, \cdots) \mathrm{V}$ &.,$\ldots 10$ & $99, \wedge r q$ & $r, r r \leq$ & $r, \vee q \mu$ & $\leq \leqslant \Lambda \uparrow$. & $\leq 11, Y 4 \leq 19$ & $0111 \leq r$ & $r \ldots$ \\
\hline.,$\ldots 1 r$ & $\cdot, \ldots 7$ & qV,TrY & $\varepsilon, 9 \vee 9$ & $11, r \leq Y$ & $\leq$ THO & $\varepsilon 1 \leq \varepsilon \cdot \leq \varepsilon \cdot r$ & $T r \cdot \leqslant 9 r$ & $r \ldots r$ \\
\hline$\cdot, \cdots r y$ & $\cdot, \ldots 1 \leq$ & $\wedge \vee, \wedge 01$ & r,น৭V & 7,179 & $\leqslant \leqslant$ rTo & $\Sigma \Sigma Y V Y \Sigma \cdot Y \wedge$ & VVIVAY & $r \ldots r$ \\
\hline.,$\ldots r v$ &.,$\ldots r r$ & Ar,qro & $Y, V Y \leq$ & $\varepsilon, T \vee Y$ & $\leq 7 \wedge \leq \Lambda$ & OYOTETYNO & $q r V V \leq 0$ & $r \ldots r$ \\
\hline$\cdot, \cdots \vee \cdot$ &,$\ldots V Y$ & $\vee \wedge, \wedge \leq 0$ & $r, \leqslant Y r$ & $r, r V I$ & $T Y 110$ & $9 . \vee(\wedge 991 \wedge$ & $|r| \leq r \ldots$ & $r \ldots \varepsilon$ \\
\hline.,$\ldots 0 \leqslant$ &.,$\cdots 91$ & $\wedge 9,7 \wedge \uparrow$ & $r, T \leq 0$ & $1, \leqslant \mu \wedge$ & 1.70 & Tororolon & 117rors & $r \ldots o$ \\
\hline.,$\ldots .07$ & $\cdot, \cdots \wedge 4$ & $1 \cdot \vee, \leqslant \wedge \leqslant$ & $1,9 \wedge 9$ & $1, Y 9 V$ & VQOYY & $V Y I \leq V Y \leq Y \wedge$ & 1. 14rvo & $r \ldots r$ \\
\hline.,$\ldots 01$ & $\cdot, \cdots \leqslant V$ & $1 \pi \cdot, \leqslant \vee q$ & 1,17 & $1, \leqslant r \wedge$ & $\varepsilon \wedge q . r$ & АVTYATq9V & $1074 \leq .9$ & $r \ldots r$ \\
\hline$\cdot, \cdot 1 \cdot V$ &.,$\ldots T r$ & $17 r, \wedge 1 \wedge$ & ג ג & $1,1 \cdot 7$ & $0 \leq 771$ & 1. Troor 1. r & rIVTArq & $r \ldots \Lambda$ \\
\hline.,$\cdot 17 \varepsilon$ & $\cdot, \cdot 1 \pi \wedge$ & $1 \wedge \wedge, 9 \wedge Y$ & $1,1 \cdot 1$ & $1, r \cdot q$ & V & $90.9099 \mathrm{M \mu}$ & $\varepsilon \varepsilon .799 \mathrm{~V}$ & $r . .9$ \\
\hline.,$\cdot 1 \vee \leqslant$ & $\cdot, \ldots \leq 0$ & $Y \backslash \wedge, \wedge \wedge \wedge$ & $\cdot, \leqslant)$ & $1,0 \mathrm{VV}$ & $0119 V$ & $1 . \wedge \leq V \leq Y M T Y$ & rq11.. & r.1. \\
\hline$\cdot, \cdot 1 \leq r$ & $\cdot, \cdots \leqslant 0$ & $r r, \ldots r$ & $\cdot, V \cdot Y$ & $r, Y 01$ & TVA & $1 r r \cdot r \mu q \cdot \Lambda \varepsilon$ & 0.94704 & $r .11$ \\
\hline.,$\cdot|V|$ & $\cdot, \ldots r q$ & rVT, ror & . r rvq & $r, Y \backslash \Lambda$ & ovar. & IrrVTV. $T \leq r$ & $\varepsilon) \varepsilon \cdot V V Y$ & $r .1 r$ \\
\hline., .111 & $\cdot, \ldots r$ & Y^7,.11 & $\cdot, \wedge \cdot V$ & Y,Aro & VTrE. & 114970749.9 & E八TVYqY & $r .1 r$ \\
\hline$\cdot, \cdots \wedge V$ & $\cdot, \ldots r$. & $r .1, \leqslant 99$ & $\cdot, \wedge \varepsilon$. & $Y, \leqslant 17$ & אוצT & 1 $\leq 1 \leq 90 \pi r 4$. & $\leqslant$ ros. T. & $r .1 \varepsilon$ \\
\hline$\cdot, \cdots \wedge \wedge$ & $\cdot, \cdots \vee v$ & $r r \cdot, \vee \vee \vee q$ & $\cdot, V \cdot O$ & $\cdot, \Lambda T V$ & 7.779 & $1 Y T \leqslant 00 . r \wedge 0$ & EYTVIA & $r .10$ \\
\hline.,$\cdot 1 \wedge r$ &,,$\cdots 9$ & qur,q & - TYO &., 009 & orरa. & IYVV $5 Y .0 Y A$ & raor. 10 & $r .17$ \\
\hline .,. &., .110 & Yro, $\Sigma$ & $\cdot, V \vee Y$ & $\cdot, 9 \cdot 9$ & TANYY & $1 \leq \cdot 1 \leq r \mid Y \wedge q$ & ב9qזrr. & r.IV \\
\hline$\cdot, \cdots 9 \leq$ &.,$\ldots 07$ & $99, \wedge \uparrow q$ & $1, \pi Y V$ & $r, 790$ & $09 Y Y \cdot .07$ & 90TTYVVYV & $r V r \cdot 711, r \mu$ & المتوسط \\
\hline
\end{tabular}

جدول V: التوزيع الجغرافى لكمية وقيمة صادرات الخرشوف الطازج إلى أهم الدول المستوردة كمتوســ للفتـرة $(r+1 \Lambda-r+19)$

\begin{tabular}{|c|c|c|c|c|}
\hline$\%$ & قيمة الصادرات (ألف دولار) & $\%$ & كمية الصادرات (طن) & الدول \\
\hline$r q, r r$ & YOs,VYA & $r q, .7$ & YIT,AYO & البحرين \\
\hline$r \wedge, 99$ & YOr, YqOV & $r \wedge, \wedge)$ & YIY, \&৭Y & إيطاليا \\
\hline 14,14 & س & $1 ., Y_{0}$ & $07,1 \ldots$ & أمريكا \\
\hline$\varepsilon, \wedge V$ & M, 1,747V & $\varepsilon, \varepsilon V$ & $r \varepsilon, \varepsilon \leqslant Y$ & لبنان \\
\hline $1,1 \mathrm{r}$ & V,rqArrr &., $9 \mathrm{~V}$ & $0, r \cdot 0$ & روسبا \\
\hline., 01 & $r, \vee 99$ &., 00 & $r, \cdot 1 r$ & السعودية \\
\hline$r, .0$ & $1 r, r \cdot T 4 V$ & 0,9 . & $r, r \cdot \varepsilon$ & دول أخرى \\
\hline $1 \ldots$ & $T \leq q, T 1 Y V$ & $1 \ldots$ & $0 \leqslant \vee, \leqslant \wedge 0$ & الإجمالى \\
\hline
\end{tabular}




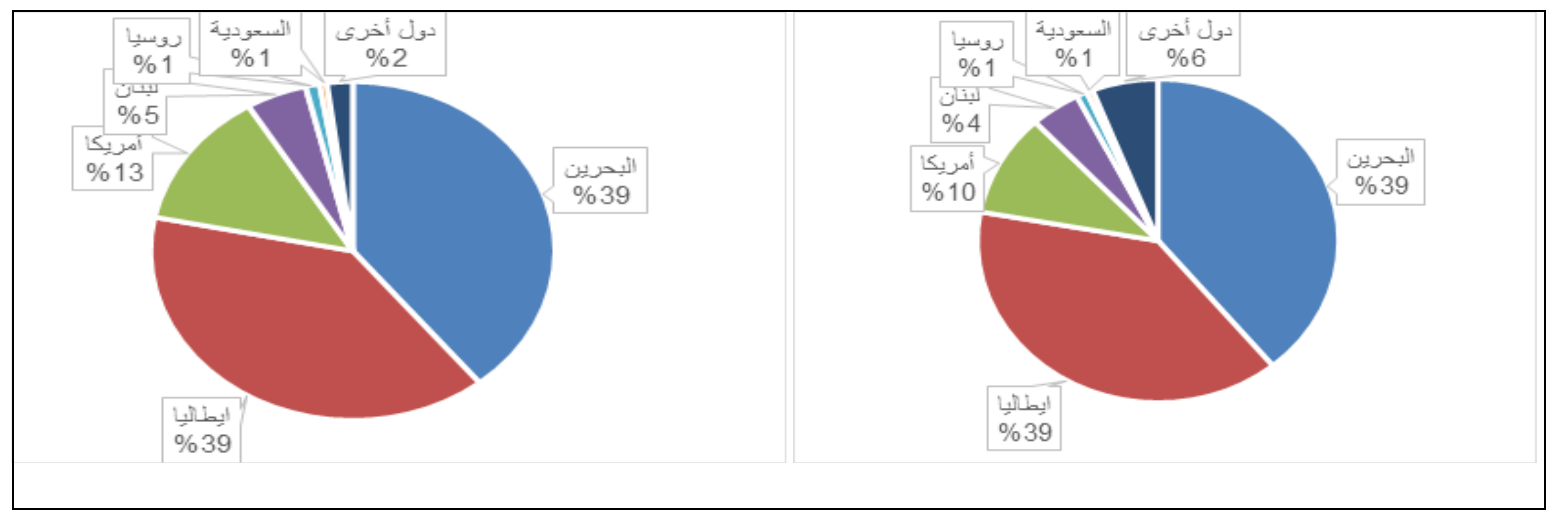

شكل ا: الأهمية النسبية لكمبة الصادرات المصـرية شكل ب: الأهمية النسبية لقيمة الصادرات المصرية من

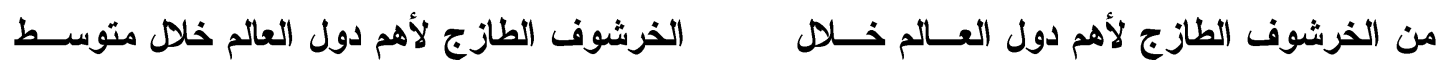

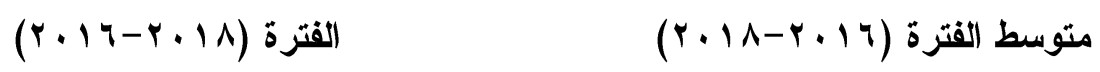

المصدر : جمعت وحسبت من الجدول (V).

جدول ^: التوزيع الجغرافي لكمية وقيمة صادرات الخرشوف المجمد إلى أهم الدول المستوردة كمتوسط للفترة

\begin{tabular}{|c|c|c|c|c|}
\hline$\%$ & قيمة الصادر ات (مليون دو لار) & $\%$ & كمية الصادر ات (ألف طن) & الدول \\
\hline$r \wedge, 79$ & $\Lambda, r Y T$ & rq, $\vee 0$ & $7,9 Y \mathrm{~V}$ & ايطاليا \\
\hline 19,17 & 0,007 & 19,19 & $\varepsilon,\{\urcorner \wedge$ & اسبانبا \\
\hline $1 \cdot, 71$ & $r, .9 V$ & 9,7 . & Y,YTO & فرنسا \\
\hline$\Lambda, r V$ & $Y, \varepsilon Y \wedge$ & $9, .0$ & $r, 1 \cdot r$ & اليونان \\
\hline$\vee, T \Lambda$ & Y,YYY & 1, Yo & $1,9 Y Y$ & اسر ائيل \\
\hline$\Lambda, \perp \vee$ & $r, r$, & $V, T)$ & $1, V \vee 1$ & بلجيكا \\
\hline$\gamma, \wedge 9$ & r,YAq & $0, V 4$ & $1, r \leqslant 1$ & أمريكا \\
\hline$\varepsilon, \lambda 1$ & 1,190 & 0,10 & $1, r \times 91$ & تركيا \\
\hline$\varepsilon, 07$ & I,Tr & $0, \cdot \varepsilon$ & $1,1 V \mu$ & دول أخرى \\
\hline $1 \ldots$ & $r q, \ldots r$ & $1 \ldots$ & $\lceil r, Y \wedge \varepsilon$ & الإجمالى \\
\hline
\end{tabular}

المصدر : حسبت من الجهاز المركزى للتعبئة العامة والاحصاء، المركز القومي للمعلومات، بيانات غير منشورة.

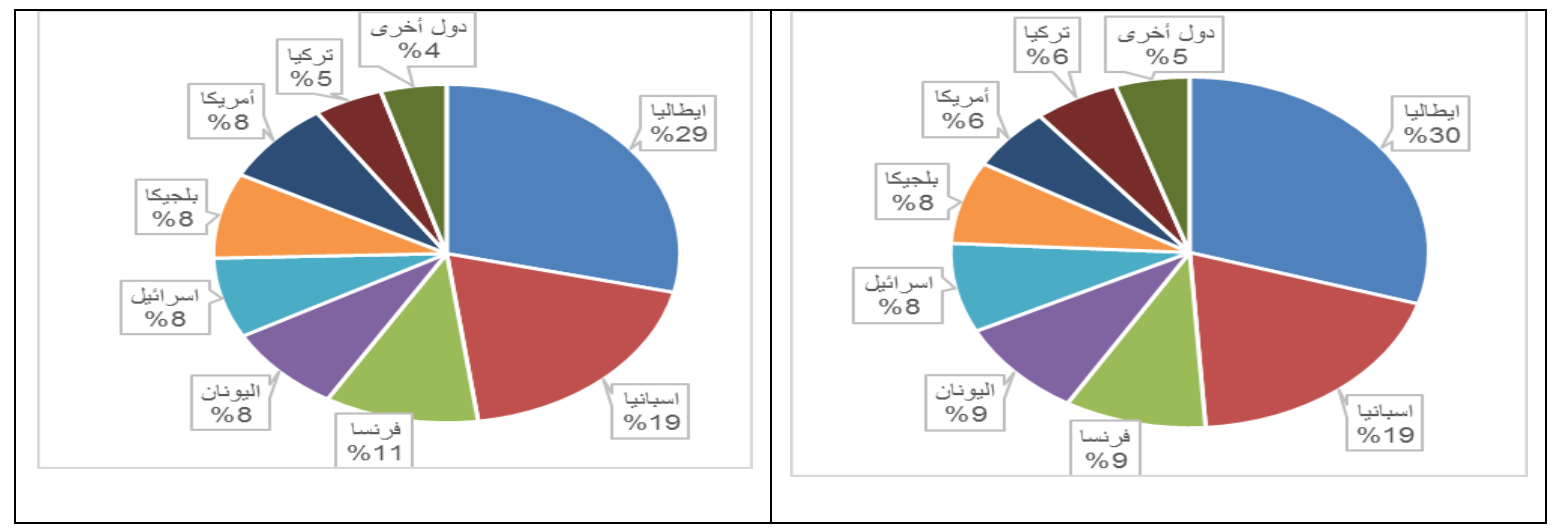

شكل rا: الأهمية النسبية لكمية الصادرات المصرية من شكل ع : الأهمية النسبية لقيمة الصادرات المصـرية من الخرشوف المجمد لأهم دول العــالم خــلال

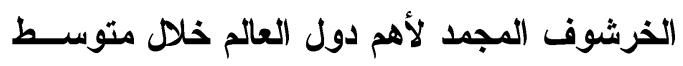

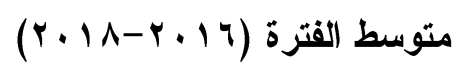

$$
\text { الفترة (1) }
$$

المصدر: جمعت وحسبت من الجدول (^). 
سعر تصدير الخرشوف من الدول المنافسة إلــى : Pj

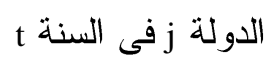

وتم إختيار أهم خمس دول مستوردة للخرشــوف المصري هي دول البحرين و إيطاليا و أمريكا وأســبانيا

وفرنسا.

نتائج تقدير نموذج المشــاركة الســوقية للخرشــوف

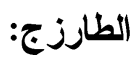

أوضحت النتائج الواردة بجــدول (9) أن قيمــة

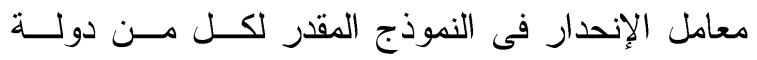

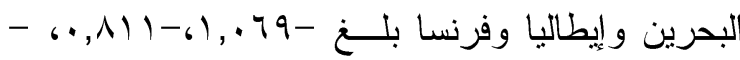

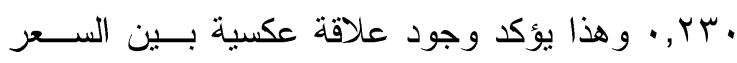
النسبى للإستير اد و النصيب السوقى من الخرشوف وهذا يتفق مع النظرية الإقتصادية. وبلغت المرونة الســعرية

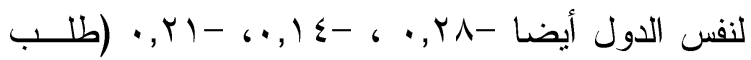

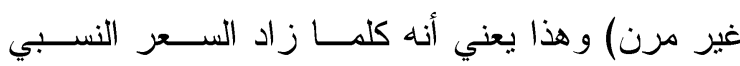
للتصدير حدث انخفاض أقل من فى النصيب الســـوقي

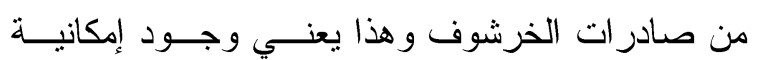
لزيادة سعر التصدير لكل من البحرين وإيطاليا وفرنسا وبالتالى زيادة العائد التصديرى للخرشوف. فى حين أوضحت النتائج الواردة بنفس الجدول أن قيمة معامل الإنحدار فى النموذج المقدر لكل من أمريكا

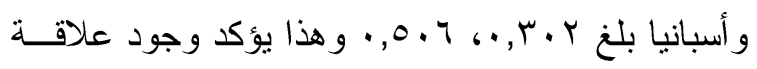

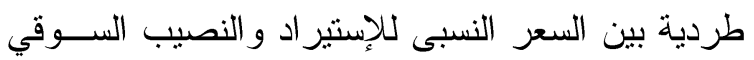

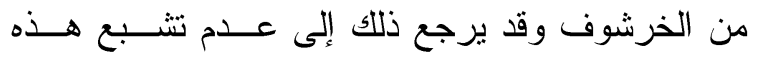
الأسواق من محصول الخرشوف وزيادة الطلب الداخلي عليها.

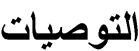

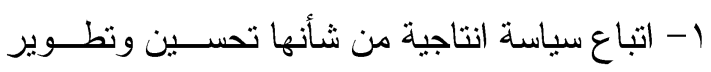
الإنتاج وخفض تكاليفه لزيادة القدرة على الوفــاء

$$
\text { بمتطلبات التصدير للأسو اق الخارجية. }
$$

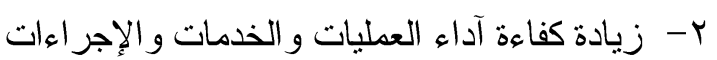

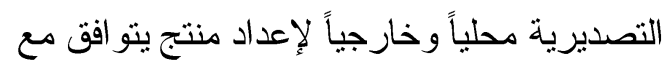

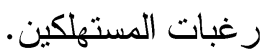

ثامنا: تقدير الطلب الخارجى على الصادرات المصرية

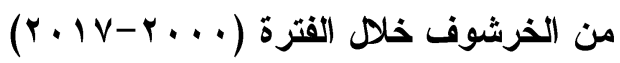
نموذج المشاركة السوقية (Market Share Model):

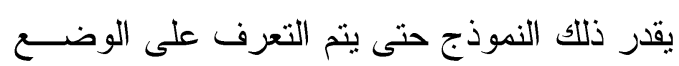

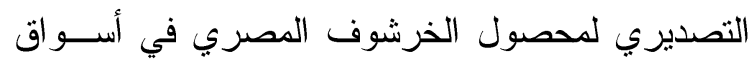
الدول المستوردة وتقدير إمكانية تتمية الوضع التتافسي

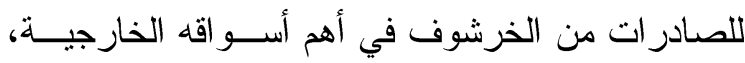

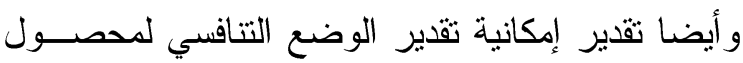
الخرشوف فى السوق الخارجي، وهو يقـيس الكميــة المصدرة من الخرشوف لدولة معينــة إلــى إجمـــالي الصادر ات من الخرشوف لجميع الدول وكذلك بالنســبة الجية للسعر فهو يقيس العلاقة بين السعر الذى تســتورد بـــه

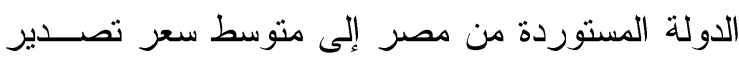

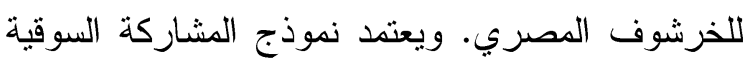
على أن النصيب السوقي للسلعة فى سوق مـــا يتــأثر بالنسبة السعرية لسعر تصدير الخرشوف المصري في لئي

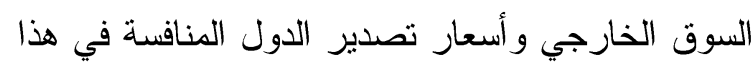
السوق ومن ثم فإن النصيب السوقى يســتخدم كمتغيـر

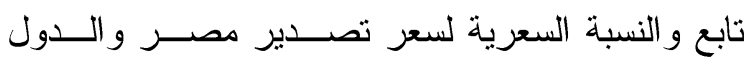
الأخرى المنافسة كمتغير ات مســتقلة (Abbot, P,1998) ويأخذ شكل النموذج الصورة النالية:

$\mathrm{Gi}=\mathrm{b}_{0}-\mathrm{b}_{1} \mathrm{P}_{1}+\mathrm{b} 2 \mathrm{G}_{\mathrm{b}-1}+\mathrm{b}_{3 \mathrm{t}}$

Gi Gi=qi/qj سابه من

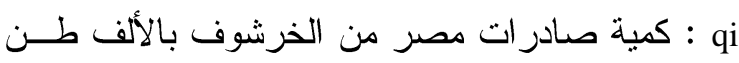
t إلى الدولة j في السنة

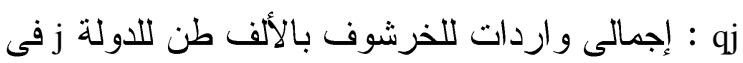
t السنة P1 الإستير اد فى الدولة الأخرى من الدولة المنافسة لهن $\mathrm{P} 1=\mathrm{Pi} / \mathrm{P}$ سعر التصدير المصرى للخرشوف بالدولار إلــى : Pi

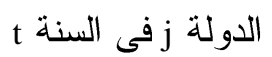


جدول 9: نتائج تقدير الطلب الخارجى على الصادرات المصرية من الخرشوف باستخدام نموذج المشاركة السوقية Market Share Model

\begin{tabular}{|c|c|c|c|c|c|c|}
\hline السرونة & $\mathbf{F}$ & R2\} $&{\text { اللصاميب السابققى }} &{\text { سعر الإستيرير اد }} &{\text { ثابت المعادلة }} &{\text { اللدولتوردة }} \\
{\hline \cdot, Y \wedge-} &{* \varepsilon, 707} &{\cdot, Y 17} &{\begin{array}{l}\cdot, 19 \leqslant \\
(\cdot, r \leqslant 0)\end{array}} &{\begin{array}{c}1, \cdot 79- \\
*(r, \wedge .1-)\end{array}} &{\begin{array}{l}r, 9 Y) \\
*(r, \wedge 9 \cdot)\end{array}} &{\text { البحرين }} \\
{\hline \cdot, 1 \leqslant-} &{* r, \vee \cdot q} &{\cdot, \mid \vee \wedge} &{\begin{array}{l}., Y 90 \\
(1, . \vee 11)\end{array}} &{\begin{array}{l}\cdot, \wedge) 1- \\
*(r, q \cdot r-)\end{array}} &{\begin{array}{c}1, \cdot \varepsilon) \\
*(r, \vee \wedge \cdot)\end{array}} &{\text { ايطاليا }} \\
{\hline \cdot, 1 T} &{r, \cdot 1 r} &{\cdot, r \cdot \mid r} &{\begin{array}{c}\cdot, r 79 \\
(1, . V 4)\end{array}} &{\begin{array}{l}\cdot, r \cdot r \\
(1, \cdot \lambda)\end{array}} &{\begin{array}{l}\cdot, T \cdot r \\
(\cdot, 9 \wedge)\end{array}} &{\text { أمريكا }} \\
{\hline \cdot, 17} &{* * q, r \circ r} &{\cdot, 701 .} &{\begin{aligned} & \cdot, 9 \wedge r \\
& * *(Y, \cdot r)\end{aligned}} &{\begin{array}{c}\cdot, 0.7 \\
* *(r, 0 \vee \Lambda)\end{array}} &{\begin{array}{l}., 100 \\
(1, .47)\end{array}} &{\text { أسبانيا }} \\
{\hline \cdot, Y)-} &{1,94 .} &{., 190} &{\begin{array}{l}\cdot, I V T \\
(\cdot, 9 r)\end{array}} &{\begin{array}{l}., Y T \cdot- \\
(1, \cdot 1-)\end{array}} &{\begin{array}{l}\cdot, r 91 \\
(1, .7)\end{array}} &{\text { فرنسا }} \\
$\hline
\end{tabular}

المصدر : جمعت وحسبت من موقع الييانات الاحصائية للنظمة الأغذية والزر اعة باستخدام برنامج النحليل الإحصائي (SPSS ver. 25).

شيماء حسن خميس حنفى،" تأثير بعض الممارسـات

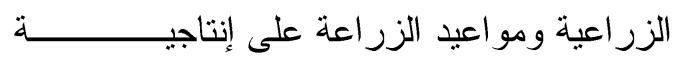

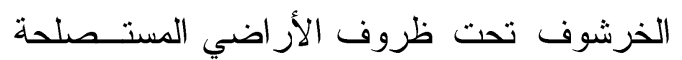

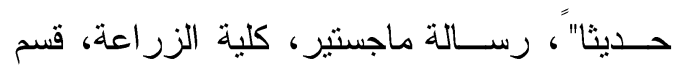

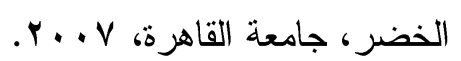

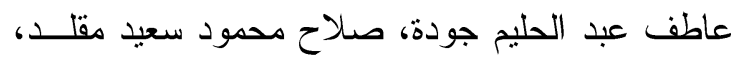

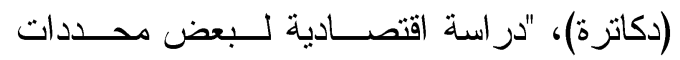

الصادرات المصرية من العنب المصري"، المجلة

المصرية للاقتصاد الزراعي، المجلد الثامن، العدد

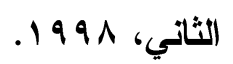

محمد رمضان إسماعيل اللقة، "المعوقات التجارية التي تو اجه الصادرات الزر اعية المصـرية"، رســالة دكتوراه، قسم الاقتصاد الزراعي، كلية الزراعة،

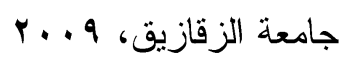

منظمة الأغذية و الزر اعة، قاعدة البيانات الاحصــائية،

$$
\text { الموقع الالكتروني www.FAO.org }
$$

Abbot, P. 1998. Elasticities in International Trade. In: Estimating U.S. Agricultural Export Demand Elasticities: Econometric and Economic Issues. Westview Press. P. 53-86.

Deardorff, A. V., Testing Trade Theories and Predicating Trade Flows, Hand boiok of International Economics, by R. W. Jones and Kenen. Amesterdam, Science publishers, 1984. Porter, M. E., Competitive Advantage of Nations, MacMillan press LDS, 1990.
ويزيد من القدرة التنافسية للصادر ات من محصول

$$
\text { الخرشوف. }
$$

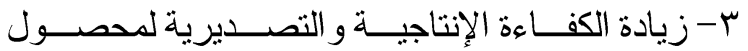

$$
\text { الخرشوف. }
$$

ـ - زيادة الاهتمام بالجو انب غير السعرية مثل الجــودة

و المو اصفات الأخرى التي تضعها الدول الرئيسـية

$$
\text { في استير اد الخرشوف. }
$$

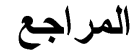

الجهاز المركزى للتعبئة العامة والإحصـــاء، المركـز القومي للمعلومات، بيانات غبر منشورة.

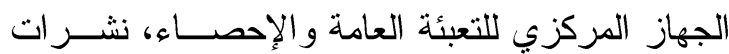
التجارة الخارجية، أعداد مختلفة. سهام داود زكـى داود، "تتافسـية اهــم الصـــادرات الزراعية المصرية في الأسواق الأوروبية، مجلة

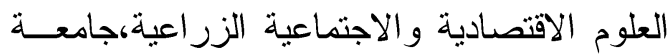

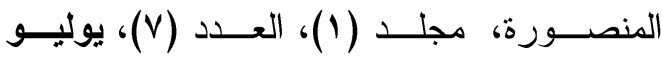
.$r \cdot 1$.

سهى مصطفى عبد العزيز الديب، "امكانيــات تتميــة الصادرات المصرية لبعض الحاصلات البســتانية

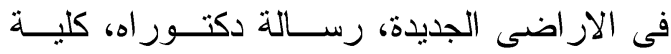
الزر اعة، جامعة عين شمس، 9 ، . . . . 


\title{
An Economic Study of Egyptian Artichoke Exports
}

\author{
Roqaya Hasan Gabr, Ruwaida Usama Owaida \\ Department of Agricultural Economics - Faculty of Agriculture - Zagazig University - Egypt
}

\begin{abstract}
This research aimed to study both the development of area, production and productivity, the development of Egyptian exports, the development of Egyptian exports, estimating indicators of economic stability, studying the geographical distribution of Egyptian exports, assessing the apparent comparative advantage of exports, measuring the efficiency of export performance, and estimating external demand for Egyptian exports of Egyptian artichoke during the period (2000-2017), The research relied on the use of methods of quantitative and descriptive analysis through various economic methods and models where time trend equations were used for the variables that were chosen and that serve the purpose of the study, and some indicators were used. Data to measure the competitiveness of exports from Egypt Artichokes such as the degree of stability and comparative advantage coefficient phenomenon and the efficiency of the performance of the export of Egyptian artichoke during the time period (2000-2017).

The most important results of the research were: The ratio of the quantity of Egyptian exports of fresh artichoke to the amount of production; it turned out to be about $10.15 \%$ during the average period (20002017), with a maximum of about $34.98 \%$ in 2004 , and a minimum of about 2.58 In 2012, with a study of the ratio of the amount of Egyptian exports from frozen artichoke to the amount of production, it turned out to be about $6.8 \%$ during the average period (2000-2017), with a maximum of about $22.59 \%$ in 2016 , and a minimum of $1.22 \%$ in 2000 , It was found that there is relative stability and stability for both the cultivated area, production and productivity with an average of about $0.21 \%, 0.68 \%$, and $0.46 \%$, respectively, during the study period, and the most stable years were These are 2014, 2008 and 2013 with a minimum of $0.62 \%, 0.13 \%$ and $0.06 \%$, respectively, and it turns out that the amount of Egyptian exports of fresh artichoke is more stable than frozen artichoke

It became clear that the Egyptian exports of fresh artichoke were concentrated in the countries of Italy, America and Libya, respectively, as the amount of Egyptian exports of frozen artichoke reached about $72 \%$ of the total amount of exports during the average of the same period. It is followed by the countries of Spain, Lebanon, Russia and Saudi Arabia, with an average quantity of about 24\% of the total quantity of Egyptian fresh artichoke, while the value of Egyptian exports of fresh Egyptian artichoke was about $91 \%$ of the total value of fresh artichoke in the countries of Italy, America and Lebanon, respectively, during the same average The period, as it became clear, is the concentration of Egyptian exports of frozen artichoke in the countries of Italy, Spain, France, Greece and Israel, respectively, where the amount of Egyptian exports of frozen artichoke reached about $75 \%$ of the total amount of exports during the average of the same period. Followed by the countries of Belgium, America, Turkey, Lebanon and Algeria, with an average quantity of about $22 \%$ of the total quantity of frozen artichoke, and the value of Egyptian exports of frozen artichoke reached about $75 \%$ of the total value of frozen artichoke in the countries of Italy, Spain, France, Greece and Belgium, respectively, during the same average Period. It is followed by the countries of Israel, America, Turkey, Lebanon and Algeria, with an average value of about $22 \%$ of the total value exported from frozen Egyptian artichoke.
\end{abstract}

Key words: economic study - exports - artichoke. 